Supporting Information (SI) for

\title{
Tuning Biosensor Cross-Reactivity Using Aptamer Mixtures
}

\author{
Yingzhu Liu, Haixiang Yu, Obtin Alkhamis, Jordan Moliver and Yi Xiao* \\ Department of Chemistry and Biochemistry, Florida International University, 11200 SW 8th \\ Street, Miami, FL, USA, 33199. \\ *Corresponding author: yxiao2@fiu.edu
}

\begin{abstract}
It is challenging to tune the response of biosensors to a set of ligands - for example, high crossreactivity to a given target family while maintaining excellent specificity against interferents - due to the lack of suitable bioreceptors. We present a novel approach for controlling the cross-reactivity of biosensors by employing defined mixtures of aptamers with differing binding properties. As a demonstration, we develop assays for specific detection of a family of illicit designer drugs, the synthetic cathinones, with customized responses to each target ligand and interferent. We first use a colorimetric dye-displacement assay to show that the binding spectra of dual-aptamer mixtures can be tuned by altering the molar ratio of these bioreceptors. Optimized assays achieve broad detection of synthetic cathinones with minimal response towards interferents, and generally demonstrate better sensing performance than assays utilizing either aptamer alone. The generality of this strategy is demonstrated with a dual-aptamer electrochemical sensor. Our approach enables customization of biosensor responsiveness to an extent that has yet to be achieved through any previously-reported aptamer engineering techniques such as sequence mutation or truncation. Since multiple aptamers for the designated target family can routinely be identified via highthroughput sequencing, we believe our strategy offers a generally applicable method for generating near-ideal aptamer biosensors for various analytical applications, including medical diagnostics, environmental monitoring, and drug detection.
\end{abstract}




\section{Table of Contents}

Materials and methods

Reagents S4

Circular dichroism S4

Isothermal titration calorimetry experiments S4

Mathermatical prediction of signal gain using a mixture of two aptamers S5

Supporting Figures and Tables S6

Table S1 DNA sequences used in this work . 66

Table S2 Aptamer, ligand, and ligand concentration used for ITC experiments and determined KDs .... S6 Figure S1 Cy7-displacement assay for MDPV detection S7

Figure S2 Chemical ctructures of synthetic cathinones and interferents used in this work ................... S8

Figure S3 Characterization of the target-binding affinity of SCA1.1 using ITC S9

Figure S4 Results of Cy7-displacement assays performed with SCA2.1 or SCA1.1 alone S10

Figure S5 Relationship between fraction of aptamer and signal gain for the Cy7-displacement assay . S11

Figure S6 Target cross-reactivity and specificity of the Cy7-displacement dual-aptamer assay ..... S12

Figure S7 Cy7-based displacement assays to detect mixtures of synthetic cathinones S13

Figure S8 PAGE analysis of Exo III digestion of SCA1.1 and SCA2.1 S14

Figure S9 PAGE analysis of SCA1.1 and SCA2.1 digestion with a mixture of Exo III and Exo I S 15

Figure S10 PAGE analysis of SCA1.1-40 and SCA2.1-40 digestion with Exo I. S16

Figure S11 Circular dichroism spectra of SCA1.1-40 and SCA2.1-40 S 16

Figure S12 Characterization of the target-binding affinity of SCA2.1-40 using ITC S17

Figure S13 Characterization of the target-binding affinity of SCA1.1-40 using ITC S18

Figure S14 Characterization of the target-binding affinity of SCA2.1-34 using ITC S19

Figure S15 Characterization of the target-binding affinity of SCA1.1-34 using ITC S20

Figure S16 SCA2.1/1.1 and SCA2.1-34/1.1-34 mixture performance in the Cy7-displacement assay . S20 Figure S17 Results of Cy7-displacement assays performed with SCA2.1-34, SCA1.1-34 or the mixture of both aptamers. $\mathrm{S} 21$

Figure S18 Performance of an E-AB sensor modified with SCA2.1-34-MB alone. S22

Figure S19 Performance of an E-AB sensor modified with SCA1.1-34-MB alone. $\mathrm{S} 23$

Figure S20 Characterization of the interferent-binding affinity of SCA1.1-34 using ITC. S24

Figure S21 Comparison of surface coverages of aptamer-modified electrodes S25

Figure S22 Squarewave voltammograms of produced using various synthetic cathinones S26 Figure S23 Squarewave voltammograms of produced using various interferents S27 
Figure S24 Experimental and predicted cross-reactivity of E-AB sensor fabricated with aptamer mixture

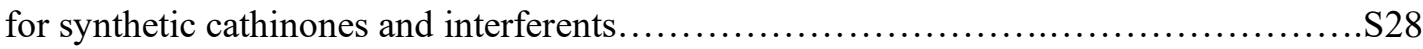

Figure S25 Squarewave voltammetry spectra for binary mixtures .............................................. S29

Figure S26 Signal gain and cross-reactivity for binary mixtures.................................................... S30

Figure S27 Cross-reactivity of aptamer mixture of SCA2.1-34 and SCA1.1-34 in the Cy7-displacement

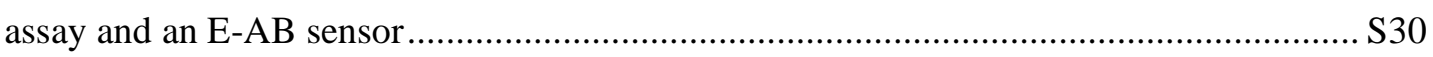




\section{Materials and methods}

Reagents. Synthetic cathinone standards (all hydrochloride salts and racemic unless specified), including 3,4-methylenedioxypyrovalerone (MDPV), 3-fluoromethcathinone (3-FMC), 4fluoromethcathinone (4-FMC), 4-methylmethcathinone (4-MMC), alphapyrrolidinopentiophenone ( $\alpha-\mathrm{PVP}$ ), butylone, cathinone, ethylone, methcathinone, methylone, naphyrone, pentylone, and pure enantiomers (-)-MDPV and (+)-MDPV were purchased from Cayman Chemicals. ( \pm )-Amphetamine hemisulfate, benzocaine, caffeine, cocaine $\mathrm{HCl}$, diethylthiatricarbocyanine iodide (Cy7), (-)-ephedrine $\mathrm{HCl}$, heroin $\mathrm{HCl}$, ketamine $\mathrm{HCl}$, lidocaine $\mathrm{HCl}$, D-lactose monohydrate, ( \pm )-3,4-methylenedioxymethamphetamine $\mathrm{HCl}$ (MDMA), Dmannitol, paracetamol, phenacetin, procaine $\mathrm{HCl},(+)$-pseudoephedrine $\mathrm{HCl}((+)-\mathrm{PSE})$, quinine hemisulfate monohydrate, tris(2-carboxyethyl)phosphine $\mathrm{HCl}$, 6-mercapto-1-hexanol (MCH), and all other chemicals were purchased from Sigma-Aldrich unless otherwise noted. Levamisole $\mathrm{HCl}$ was purchased from MP Biomedical. Sucrose was purchased from Amresco. Exonuclease III ( $E$. coli) and exonuclease I (E. coli) were purchased from New England Biolabs. SYBR Gold was purchased from Thermo Fisher Scientific. Deionized water (resistivity of $18.2 \mathrm{M} \Omega \mathrm{cm}$ ) was produced using a Milli-Q Direct 8 Water Purification System. Unmodified oligonucleotides were synthesized by Integrated DNA Technologies with HPLC purification and dissolved in PCR-grade water. Thiolated methylene-blue-modified oligonucleotides were purchased from LGC Biosearch Technologies with HPLC purification and dissolved in $10 \mathrm{mM}$ Tris- $\mathrm{HCl}(\mathrm{pH}$ 8.0) with $1 \mathrm{mM}$ EDTA. DNA concentrations were measured using a NanoDrop 2000 (Thermo Fisher Scientific). All DNA sequences used in this work are listed in Table S1.

Circular dichroism (CD). Experiments were performed in reaction buffer (10 mM Tris-HCl, 20 $\mathrm{mM} \mathrm{NaCl}, 0.5 \mathrm{mM} \mathrm{MgCl}_{2}, \mathrm{pH}$ 7.4). Before each experiment, the reaction buffer was degassed for 15 minutes using an ultrasonic cleaner (Fisher Scientific FS60D). SCA1.1-40 or SCA2.1-40 (final concentration $1 \mu \mathrm{M}$ ) dissolved in this degassed buffer was heated at $95{ }^{\circ} \mathrm{C}$ for 10 minutes and immediately cooled down on ice. $\mathrm{MgCl}_{2}$ and $\mathrm{NaCl}$ (final concentration of $0.5 \mathrm{mM}$ and $20 \mathrm{mM}$, respectively) and MDPV (racemic, final concentration $100 \mu \mathrm{M}$ ) were added to the aptamer solution and incubated overnight at room temperature. $300 \mu \mathrm{L}$ of each sample was added into a quartz cuvette (Hellma Analytics) and the CD spectra were recorded from 220-300 nm using a spectropolarimeter (JASCO J-815) with the following parameters: 5 mdeg sensitivity, $0.2 \mathrm{~nm}$ data pitch, $50 \mathrm{~nm} /$ minute scanning speed, $1 \mathrm{~nm}$ bandwidth, and 8 seconds response time. Each sample was scanned four times, and the data were averaged. Spectra of the reaction buffer, reaction buffer containing $100 \mu \mathrm{M} \mathrm{MDPV}$, and reaction buffer containing $1 \mu \mathrm{M}$ aptamer were also obtained. All CD spectra were corrected by subtracting the spectra of the buffer with and without MDPV.

Isothermal titration calorimetry (ITC) experiments. All ITC experiments were carried out at $23{ }^{\circ} \mathrm{C}$ using a MicroCal iTC200 Instrument (Malvern). For each experiment, $300 \mu \mathrm{L}$ of an aptamer solution containing $20 \mu \mathrm{M}$ of SCA1.1, SCA1.1-40 or SCA2.1-40 in reaction buffer (10 mM Tris$\mathrm{HCl}, 20 \mathrm{mM} \mathrm{NaCl}, 0.5 \mathrm{mM} \mathrm{MgCl}_{2}, \mathrm{pH}$ 7.4) were heated at $95^{\circ} \mathrm{C}$ for 10 minutes and immediately 
cooled down on ice before loading into the sample cell. The syringe was then loaded with (-)MDPV, (+)-MDPV, MDMA, (+)-PSE, procaine, levamisole, or quinine prepared in the same buffer. Since MDPV is a chiral compound, ITC experiments for the binding affinity of MDPV towards the aptamers was performed with each enantiomer individually. Concentrations of aptamers and ligands are listed in Table S2. Each experiment consisted of an initial purge injection of $0.4 \mu \mathrm{L}$ and 19 successive injections of $2 \mu \mathrm{L}$, with a spacing of $180 \mathrm{sec}$ between adjacent injections. For (+)-PSE, procaine, and levamisole, 20 more injections were performed following these 20 injections, with an initial purge injection of $0.4 \mu \mathrm{L}$ and 19 successive injections of $2 \mu \mathrm{L}$. The obtained raw data were first corrected with dilution heat obtained from ligand-to-buffer titrations and then analyzed using the MicroCal analysis kit integrated into Origin 7 software, with a single-site binding model to calculate dissociation constants $\left(\mathrm{K}_{\mathrm{Ds}}\right)$.

Mathematical prediction of signal gain using a mixture of two aptamers. A ligand binding to an aptamer with 1:1 stoichiometry follows the Langmuir equation:

$$
\left.\theta=\frac{K \cdot L}{K \cdot L+1} \quad \text { (Eq. } 1\right)
$$

Where $\theta$ is the fraction occupancy of the aptamer, $L$ is the concentration of the free ligand, and $K$ is the association constant of the aptamer. For the sensor platforms presented in this work, the signal gain is proportional to the aptamer occupancy, therefore:

$$
S=\frac{K \cdot L}{K \cdot L+1} \cdot S_{M}
$$

where $S$ is the signal gain produced by the aptamer with a given ligand concentration, and $S_{M}$ is the maximum signal gain achieved when the aptamer is completely saturated by the ligand. When a mixture of two aptamers is used in a single biosensor, both of which bind the ligand independently, the binding curve can be expressed by the following equation:

$$
\theta_{\text {app }}=\alpha \cdot\left(\frac{K_{1} \cdot L}{K_{1} \cdot L+1}\right)+(1-\alpha) \cdot\left(\frac{K_{2} \cdot L}{K_{2} \cdot L+1}\right)
$$

where $\theta_{\text {app }}$ is the global fraction occupancy of the aptamer mixture, $K_{1}$ and $K_{2}$ are the association constants of aptamer 1 and aptamer 2, respectively, and $\alpha$ is the molar fraction of aptamer 1 in the mixture. Therefore, the signal gain at a given ligand concentration can be calculated as:

$$
S_{a p p}=\alpha \cdot S_{M 1} \cdot\left(\frac{K_{1} \cdot L}{K_{1} \cdot L+1}\right)+(1-\alpha) \cdot S_{M 2} \cdot\left(\frac{K_{2} \cdot L}{K_{2} \cdot L+1}\right)
$$

where $S_{a p p}$ is the signal gain produced by the aptamer mixture, and $S_{M 1}$ and $S_{M 2}$ are the maximum signal gain respectively achieved by aptamer 1 and aptamer 2 when each aptamer is completely saturated by the ligand. Another expression of the equation is:

$$
S_{a p p}=-\left[\left(\frac{K_{1} \cdot L}{K_{1} \cdot L+1}\right) \cdot S_{1}+\left(\frac{K_{2} \cdot L}{K_{2} \cdot L+1}\right) \cdot S_{2}\right] \cdot \alpha+S_{2} \cdot\left(\frac{K_{2} \cdot L}{K_{2} \cdot L+1}\right)
$$

Thus, for a given ligand concentration and a fixed total concentration of aptamers, the signal gain is linearly related to the molar fraction of aptamer 1 .

\section{References:}

[1] W. Yang, H. Yu, O. Alkhamis, Y. Liu, J. Canoura, F. Fu, Y. Xiao, Nucleic Acids Res. 2019, 47, e71. 
[2] J. Canoura, Z. Wang, H. Yu, O. Alkhamis, F. Fu, Y. Xiao, J. Am. Chem. Soc. 2018, 140, 9961-9971.

[3] Y. Xiao, R. Y. Lai, K. W. Plaxco, Nat. Protoc. 2007, 2, 2875-80.

[4] T. M. Herne, M. J. Tarlov, J. Am. Chem. Soc. 1997, 119, 8916-8920.

Table S1. DNA sequences used in this work.

\begin{tabular}{|l|l|}
\hline Sequence ID & Sequence $\left(\mathbf{5}^{\prime} \mathbf{-} \mathbf{3}^{\prime}\right.$ ) \\
\hline SCA2.1 & CTTACGACCTTAAGTGGGGTTCGGGTGGAGTTTATGGGGTCGTAAG \\
\hline SCA2.1-40 & CTTACGACCTTAAGTGGGGTTCGGGTGGAGTTTATGGGGT \\
\hline SCA2.1-34 & ACCTTAAGTGGGGTTCGGGTGGAGTTTATGGGGT \\
\hline SCA1.1 & CTTACGACTGAGAAGTGTGATTCAGTATGTTTTCCGAGTCGTAAG \\
\hline SCA1.1-40 & CTTACGACTGAGAAGTGTGATTCAGTATGTTTTCCGAAGT \\
\hline SCA1.1-34 & ACTGAGAAGTGTGATTCAGTATGTTTTCCGAAGT \\
\hline SCA-2.1-34-MB & SH-C6-ACCTTAAGTGGGGTTCGGGTGGAGTTTATGGGGT-MB \\
\hline SCA-1.1-34-MB & SH-C6-ACTGAGAAGTGTGATTCAGTATGTTTTCCGAAGT-MB \\
\hline
\end{tabular}

$\mathrm{SH}=$ thiol modification; $\mathrm{MB}=$ methylene blue modification; $\mathrm{C} 6=\left(\mathrm{CH}_{2}\right)_{6}$ linker

Table S2. Aptamer, ligand, and ligand concentration used for ITC experiments and determined $\mathrm{K}_{\mathrm{D}} \mathrm{S}$.

\begin{tabular}{|c|c|c|c|}
\hline Aptamer & Ligand & Ligand concentration $(\mu \mathrm{M})$ & Dissociation constant $(\mu \mathrm{M})$ \\
\hline SCA1.1 & (-)-MDPV & 350 & $0.23 \pm 0.02$ \\
\hline SCA1.1 & (+)-MDPV & 1,000 & $30.4 \pm 1.0$ \\
\hline SCA1.1-40 & (-)-MDPV & 400 & $1.05 \pm 0.05$ \\
\hline SCA1.1-40 & (+)-MDPV & 2,000 & $181 \pm 2$ \\
\hline SCA1.1-34 & (-)-MDPV & 400 & $1.46 \pm 0.03$ \\
\hline SCA1.1-34 & (+)-MDPV & 2,000 & $185 \pm 3$ \\
\hline SCA2.1 & (-)-MDPV & 225 & $0.047 \pm 0.0075$ \\
\hline SCA2.1 & (+)-MDPV & 400 & $3.61 \pm 0.12$ \\
\hline SCA2.1-40 & (-)-MDPV & 400 & $0.65 \pm 0.03$ \\
\hline SCA2.1-40 & (+)-MDPV & 2,000 & $292 \pm 14$ \\
\hline SCA2.1-34 & (-)-MDPV & 400 & $0.30 \pm 0.04$ \\
\hline SCA2.1-34 & (+)-MDPV & 2,000 & $96 \pm 4.0$ \\
\hline
\end{tabular}




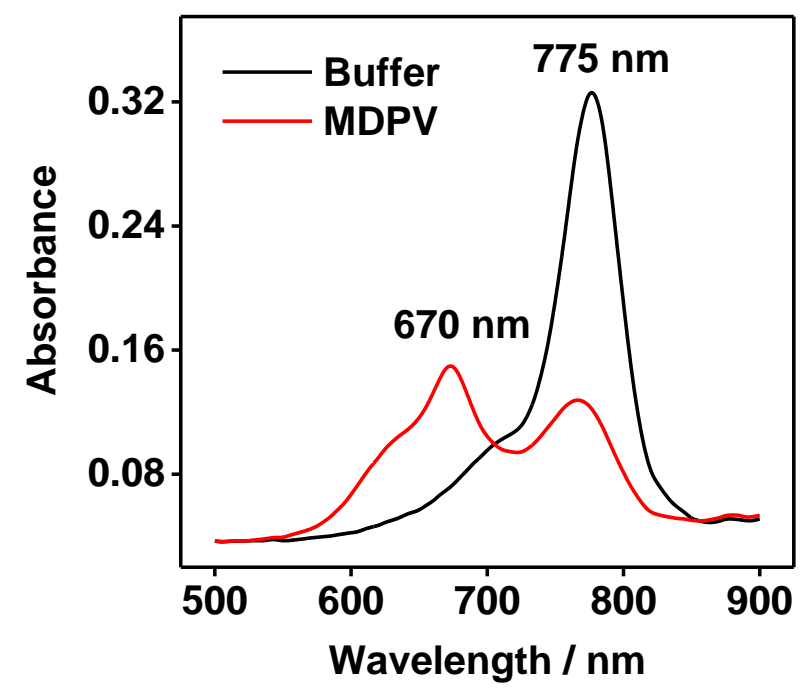

Figure S1. Cy7-displacement assay for MDPV detection. Absorbance spectra of $2 \mu \mathrm{M}$ Cy7 and 3 $\mu \mathrm{M}$ SCA2.1 in the absence (black) or presence (red) of $10 \mu \mathrm{M}$ MDPV. 

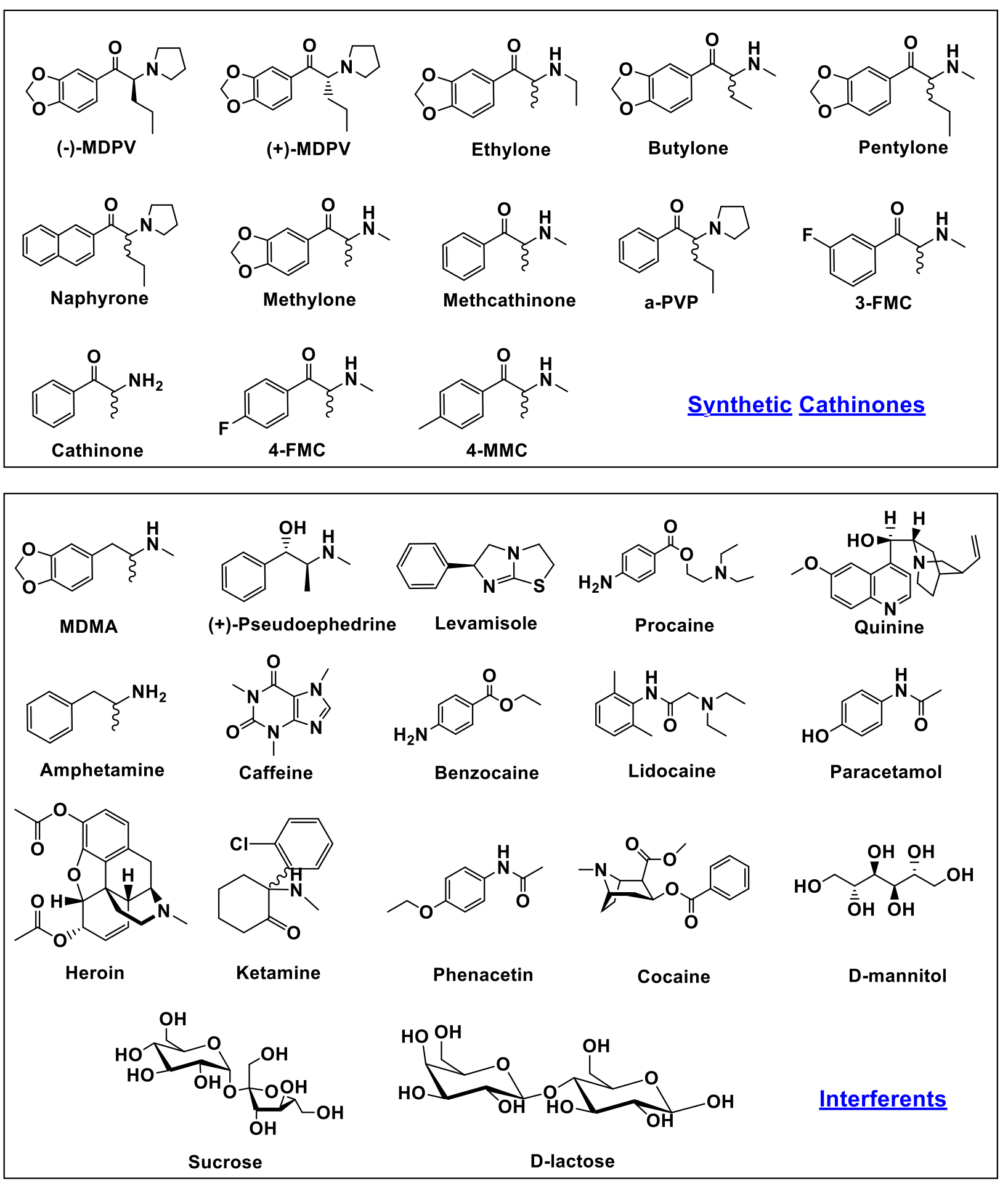

Figure S2. Chemical structures of different synthetic cathinones and interferents used in this work. 

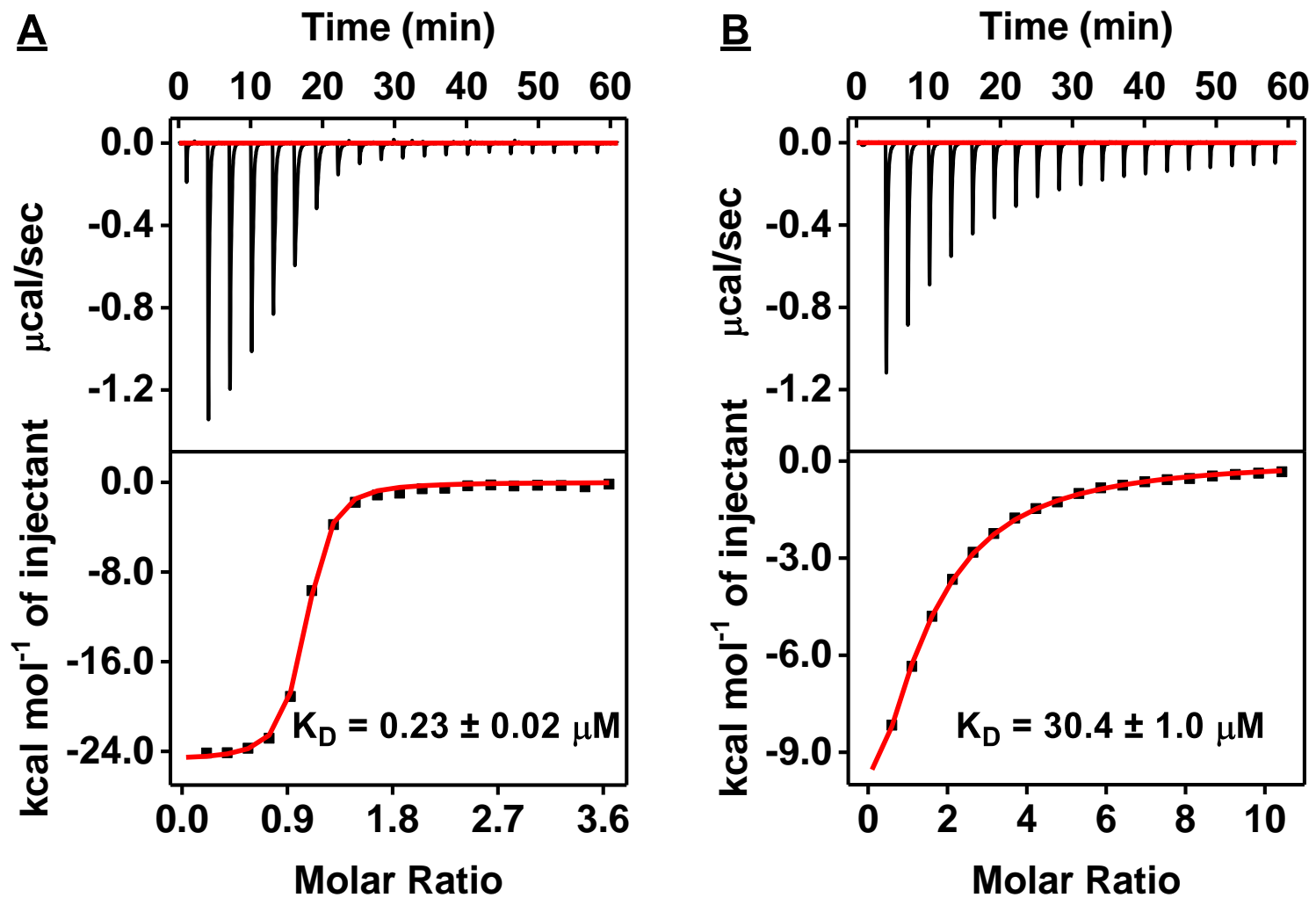

Figure S3. Characterization of the target-binding affinity of SCA1.1 using ITC. Top panels present raw data showing the heat generated from each titration of (A) (-)-MDPV and (B) (+)-MDPV into SCA1.1. Bottom panels show the integrated heat of each titration after correcting for dilution heat of the titrant. ITC data were fitted using a single-site model. 


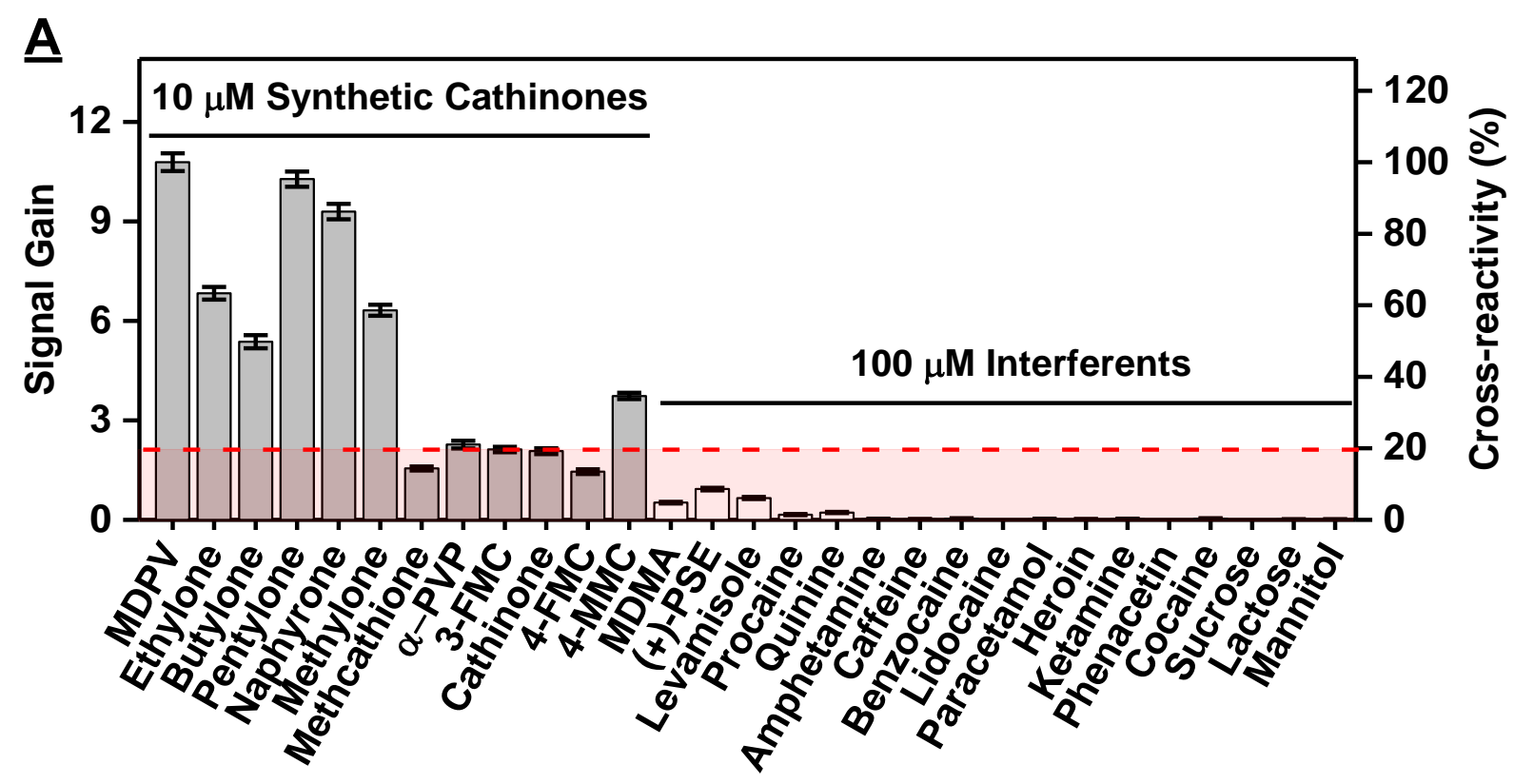

B

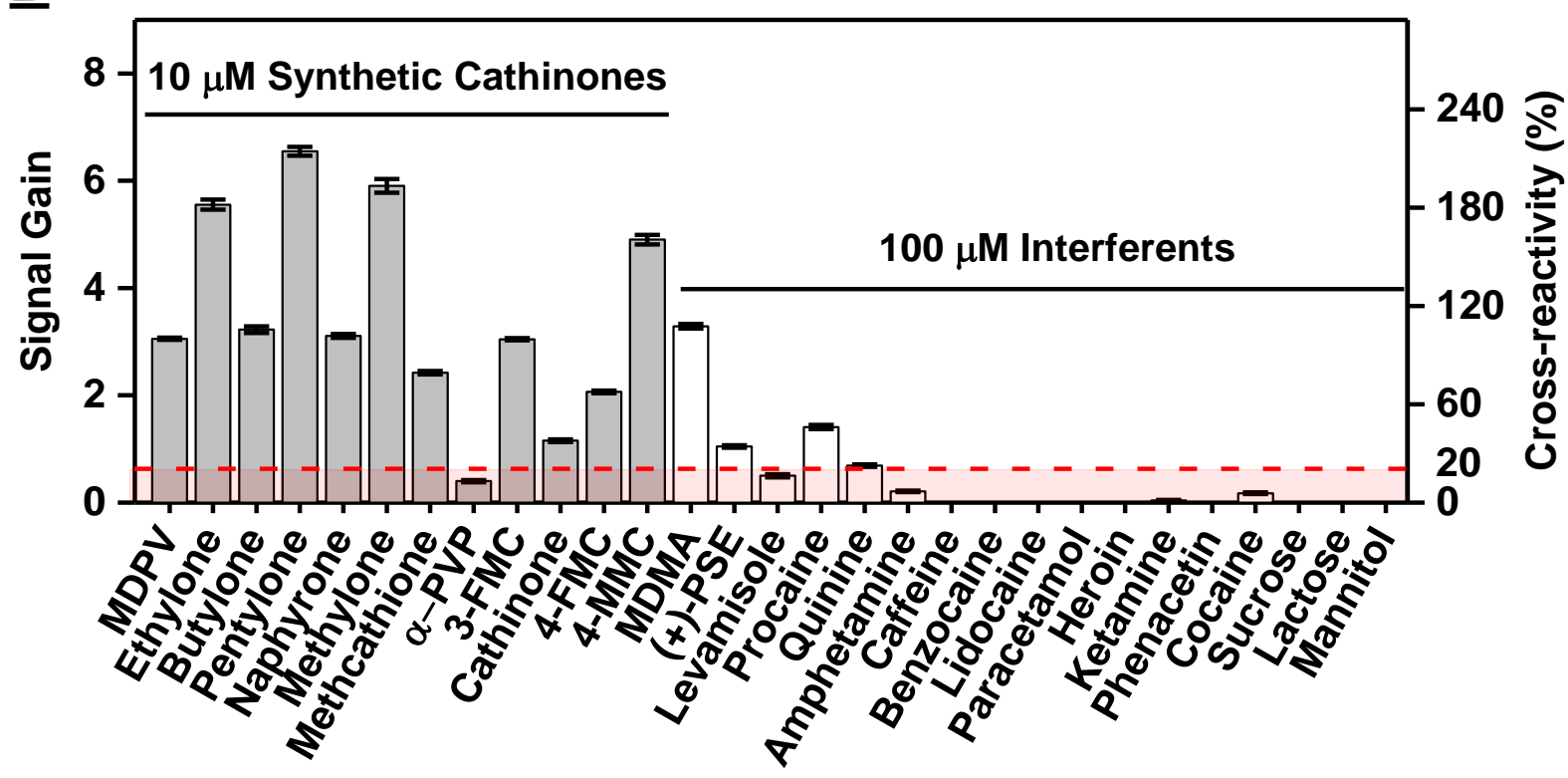

Figure S4. Signal gain and cross-reactivity from colorimetric Cy7-displacement assays performed with (A) SCA2.1 or (B) SCA1.1 against 12 synthetic cathinones (gray) at a concentration of 10 $\mu \mathrm{M}$ and 17 interferents (white) at a concentration of $100 \mu \mathrm{M}$. The cut-off for $20 \%$ cross-reactivity is marked in red. Final concentrations of aptamer and Cy7 in each experiment were $3 \mu \mathrm{M}$ and 2 $\mu \mathrm{M}$, respectively. Error bars represent the standard deviation of three measurements. 
$\underline{\mathbf{A}}$

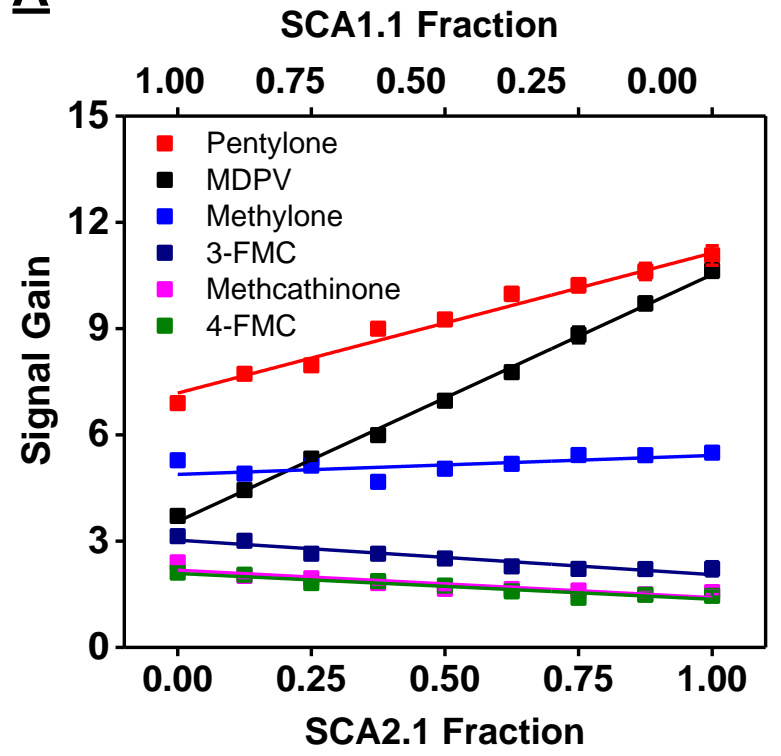

$\underline{B}$

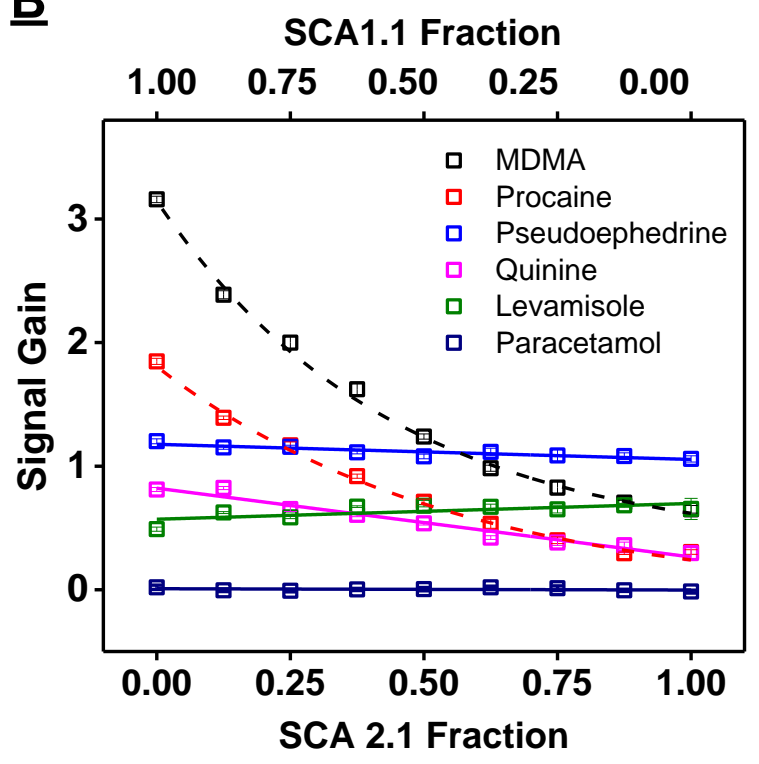

Figure S5. The relationship between the fraction of SCA2.1/SCA1.1 in the aptamer mixture and signal gain obtained for various (A) synthetic cathinones or (B) interferents in the Cy7displacement dual-aptamer assay. The total aptamer concentration was $3 \mu \mathrm{M}$ for each experiment. Error bars represent the standard deviation of three measurements. 

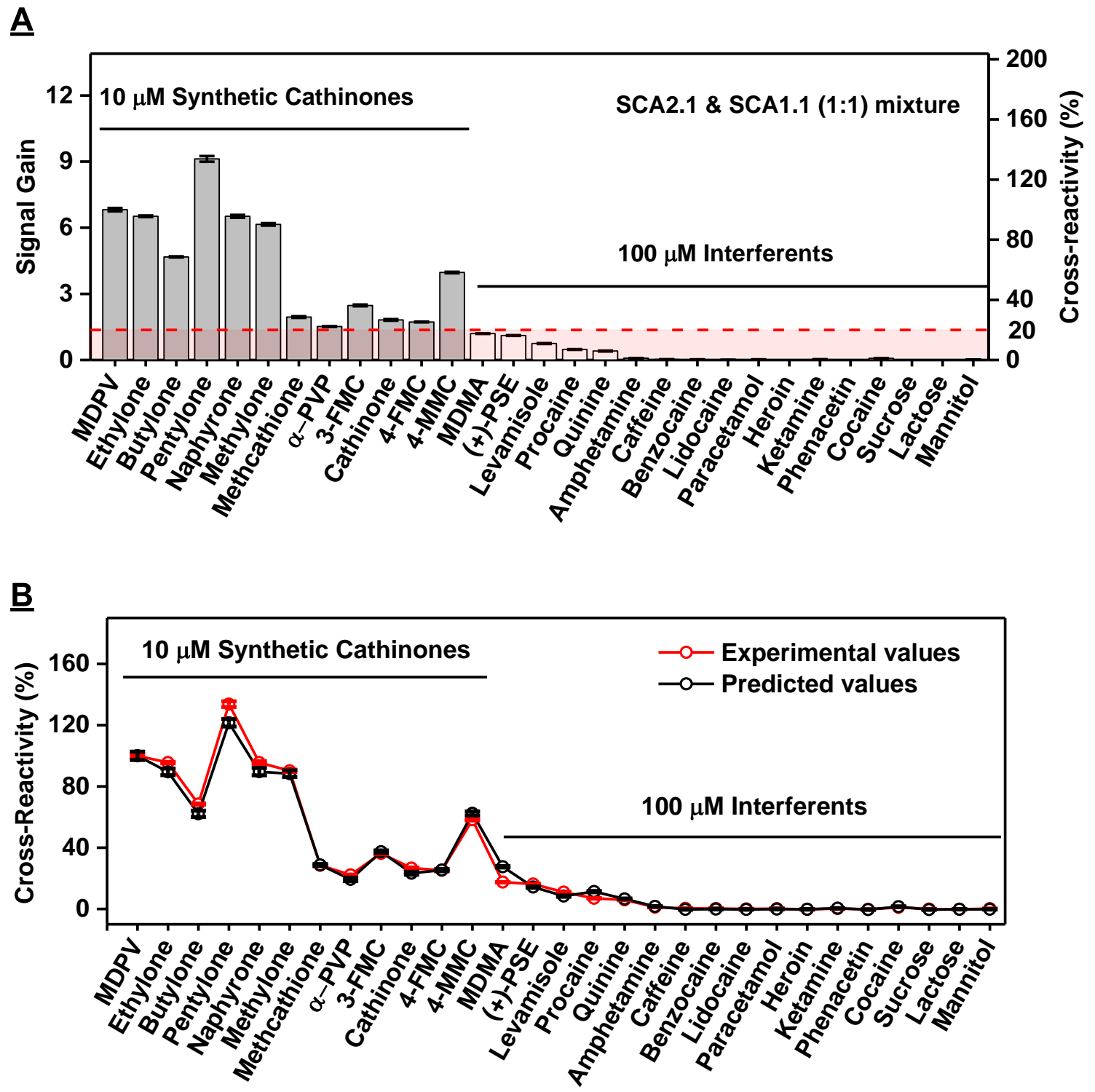

Figure S6. Target cross-reactivity and specificity of the Cy7-displacement dual-aptamer assay. (A) High target cross-reactivity and specificity were achieved when these aptamers were present at a 1:1 ratio (total aptamer concentration was $3 \mu \mathrm{M}$ ). (B) The predicted cross-reactivity (black) values for this 1:1 mixture, which we calculated from the signal gain achieved from SCA2.1 alone and SCA1.1 alone, is similar to that obtained experimentally (red) for both targets and interferents. Cross-reactivity was calculated relative to the signal gain produced by MDPV. 
$\underline{\mathbf{A}}$

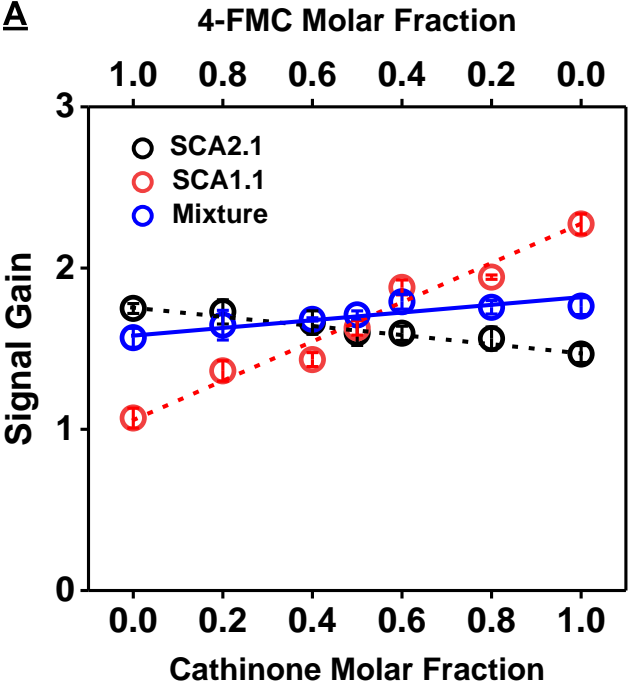

B $\quad$ Methylone Molar Fraction

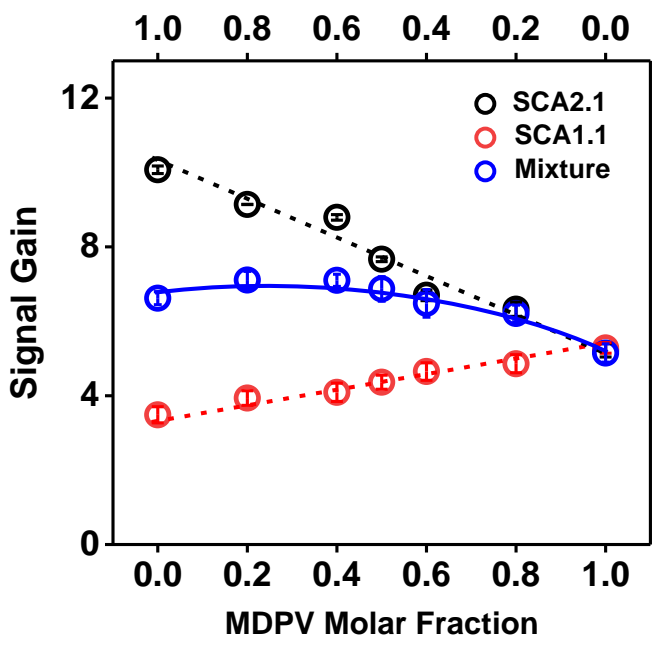

Figure S7. Cy7-based displacement assays using SCA2.1, SCA1.1, and a mixture of both aptamers to detect different mixtures of synthetic cathinones. Signal gain is shown at different molar fractions of (A) 4-FMC + cathinone and (B) methylone + MDPV. The total target concentration for each mixture is $10 \mu \mathrm{M}$. Black, red, and blue represent the results from SCA2.1, SCA1.1, and an equimolar mixture of both, respectively. Error bars represent the standard deviation of measurements from two different sets of experiments. For single aptamer experiments, $3 \mu \mathrm{M}$ aptamer and $2 \mu \mathrm{M} \mathrm{Cy} 7$ were used. For aptamer mixture experiments, $1.5 \mu \mathrm{M}$ of each aptamer was used along with $2 \mu \mathrm{M}$ Cy7. 

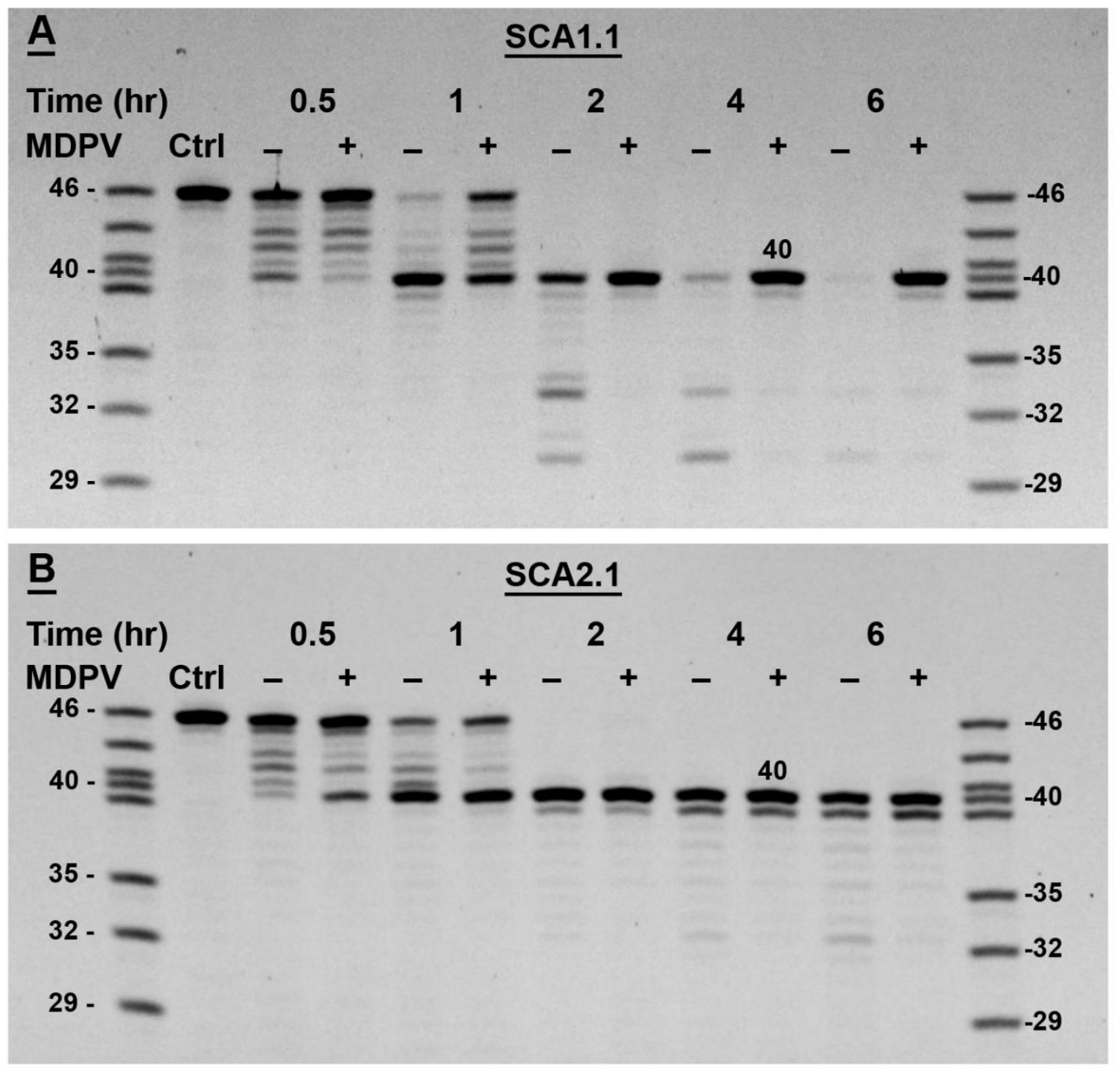

Figure S8. PAGE analysis of time-course digestion of (A) SCA1.1 and (B) SCA2.1 incubated with Exo III over 6 hours in the absence (-) and presence (+) of $250 \mu \mathrm{M}$ MDPV. Ctrl (control) contains the same concentration of aptamer with no enzyme added. 

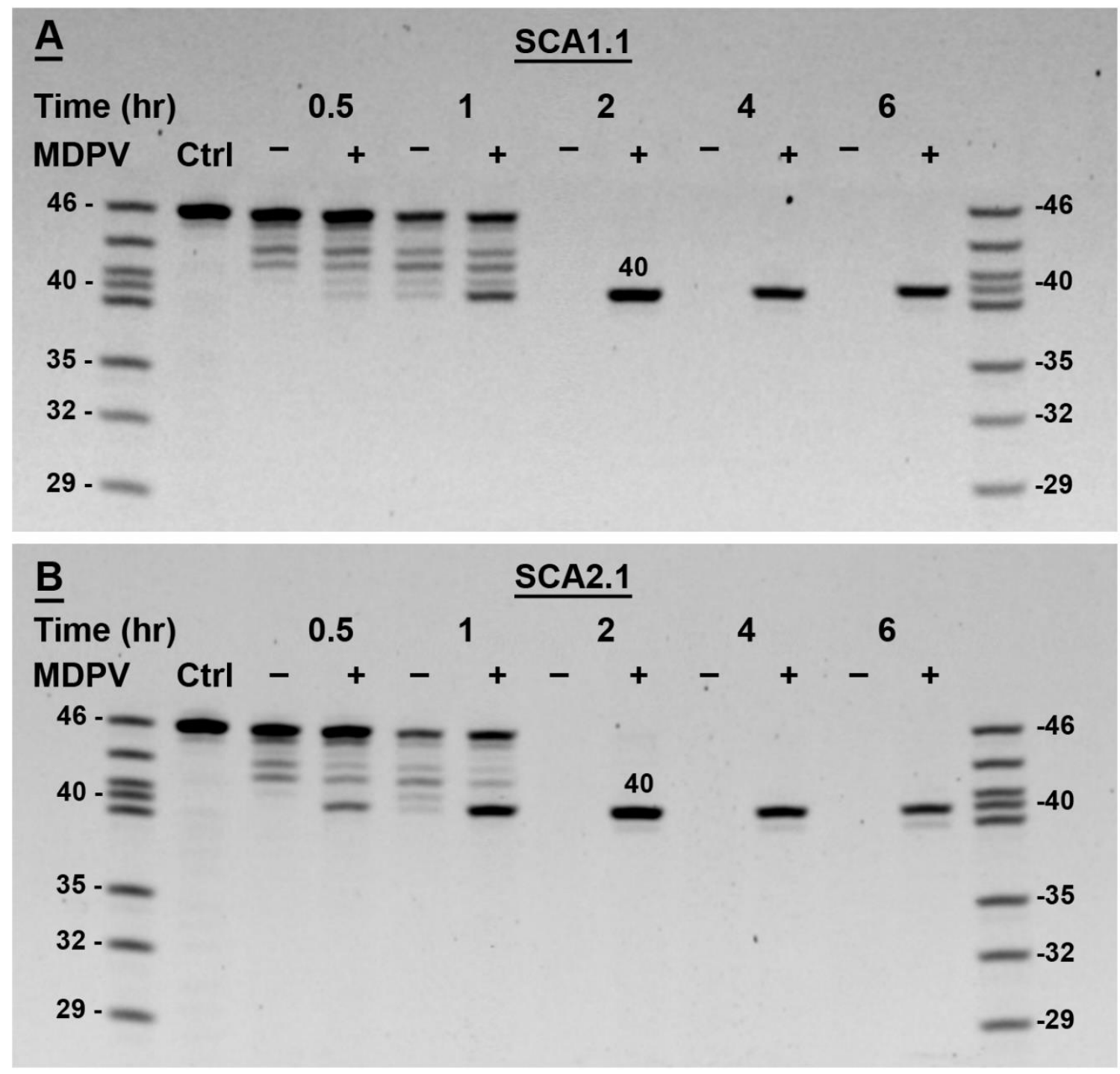

Figure S9. PAGE analysis of time-course digestion of (A) SCA1.1 and (B) SCA2.1 incubated with a mixture of Exo III and Exo I over 6 hours in the absence (-) and presence (+) of $250 \mu \mathrm{M}$ MDPV. Ctrl (control) contains the same concentration of aptamer with no enzyme added. 


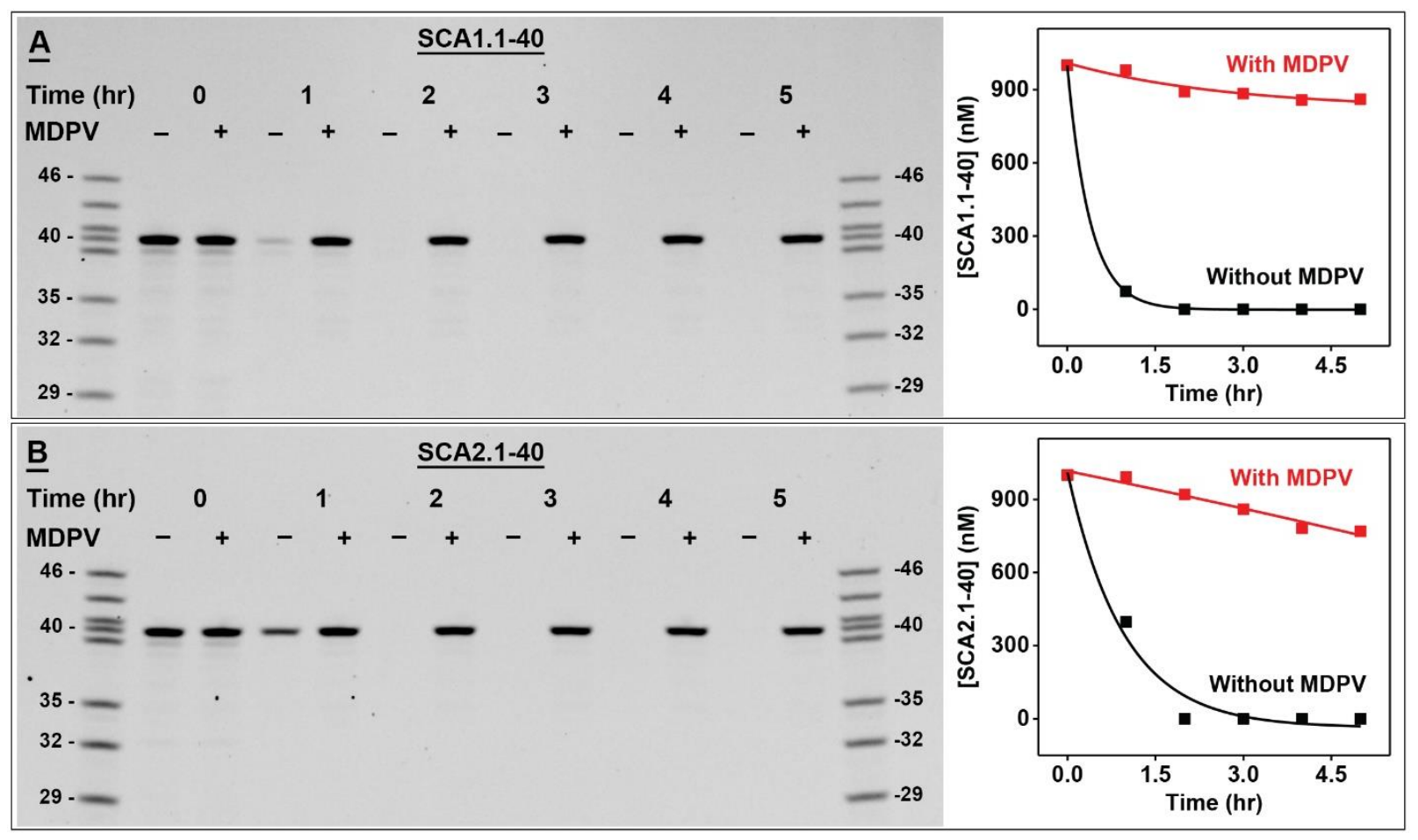

Figure S10. PAGE analysis of time-course digestion of (A) SCA1.1-40 and (B) SCA2.1-40 by Exo I over 5 hours in the absence (-) and presence (+) of $250 \mu \mathrm{M}$ MDPV. Plots at right show changes in the concentration of intact 40-nt aptamer relative to the 40-nt ladder band over time.
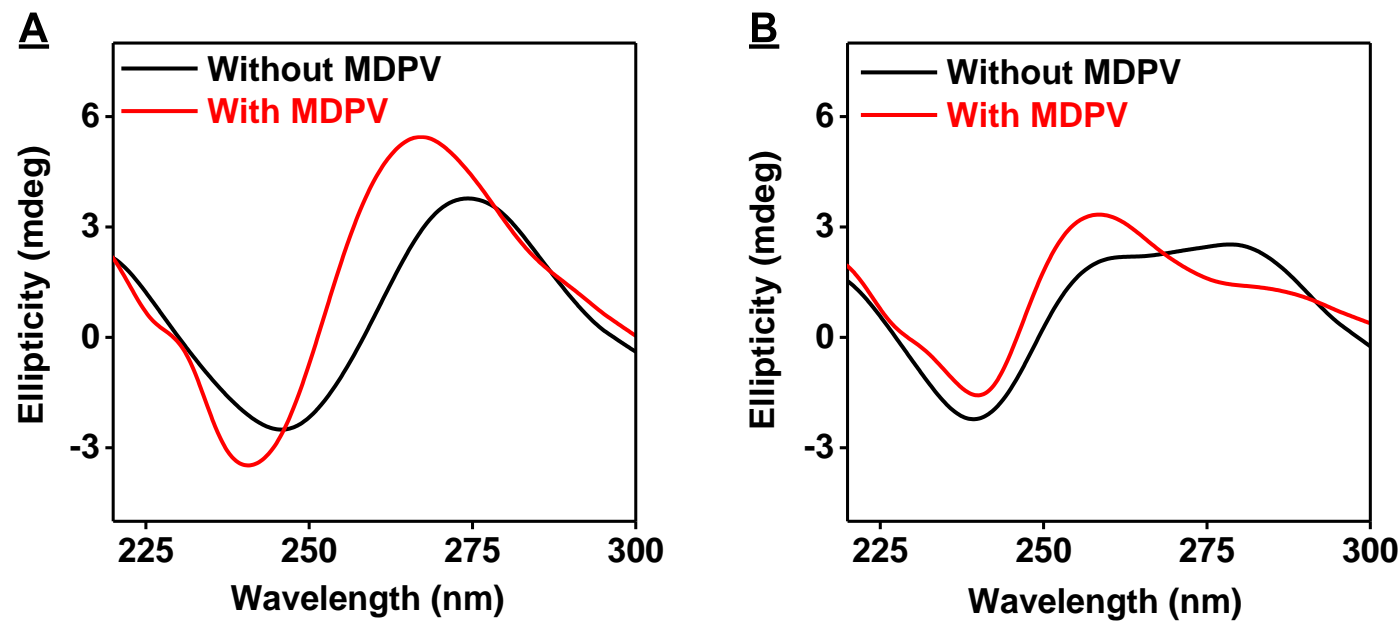

Figure S11. CD spectra of (A) SCA1.1-40 and (B) SCA2.1-40 in the absence (black) and presence (red) of $100 \mu \mathrm{M}$ MDPV after subtracting spectra of reaction buffer without or with MDPV. 
$\underline{A}$

Time $(\min )$

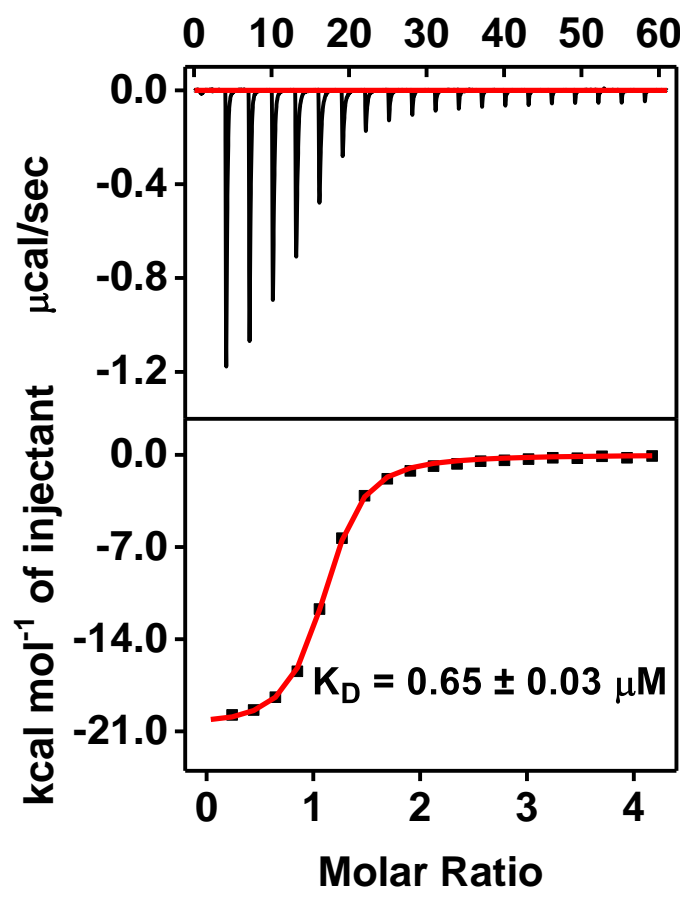

$\underline{B}$

Time ( $\min )$

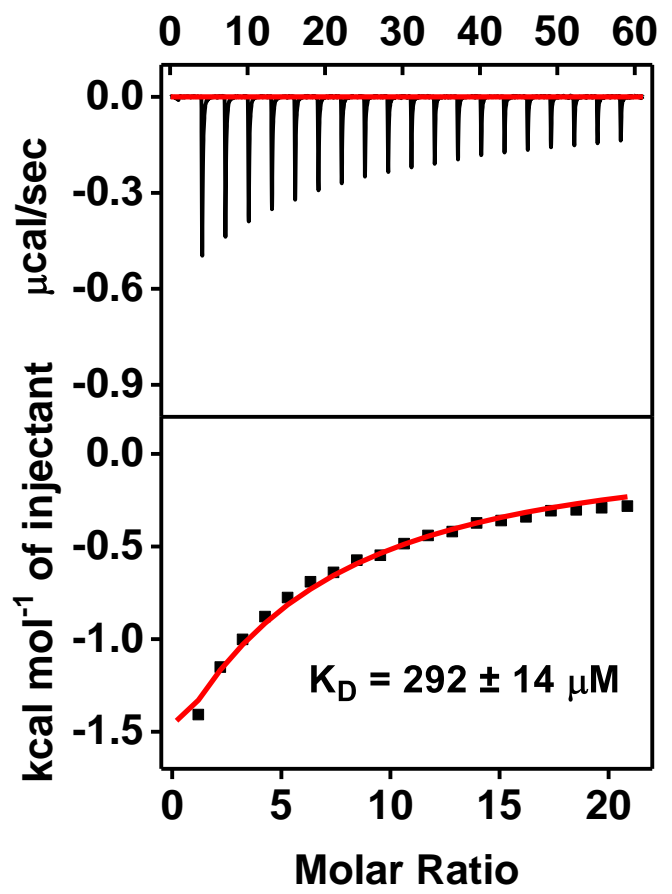

Figure S12. Characterization of the target-binding affinity of SCA2.1-40 using ITC. Top panels present raw data showing the heat generated from each titration of (A) (-)-MDPV and (B) (+)MDPV to SCA2.1-40, while bottom panels show the integrated heat of each titration after correcting for dilution heat of the titrant. ITC data were fitted using a single-site model. 

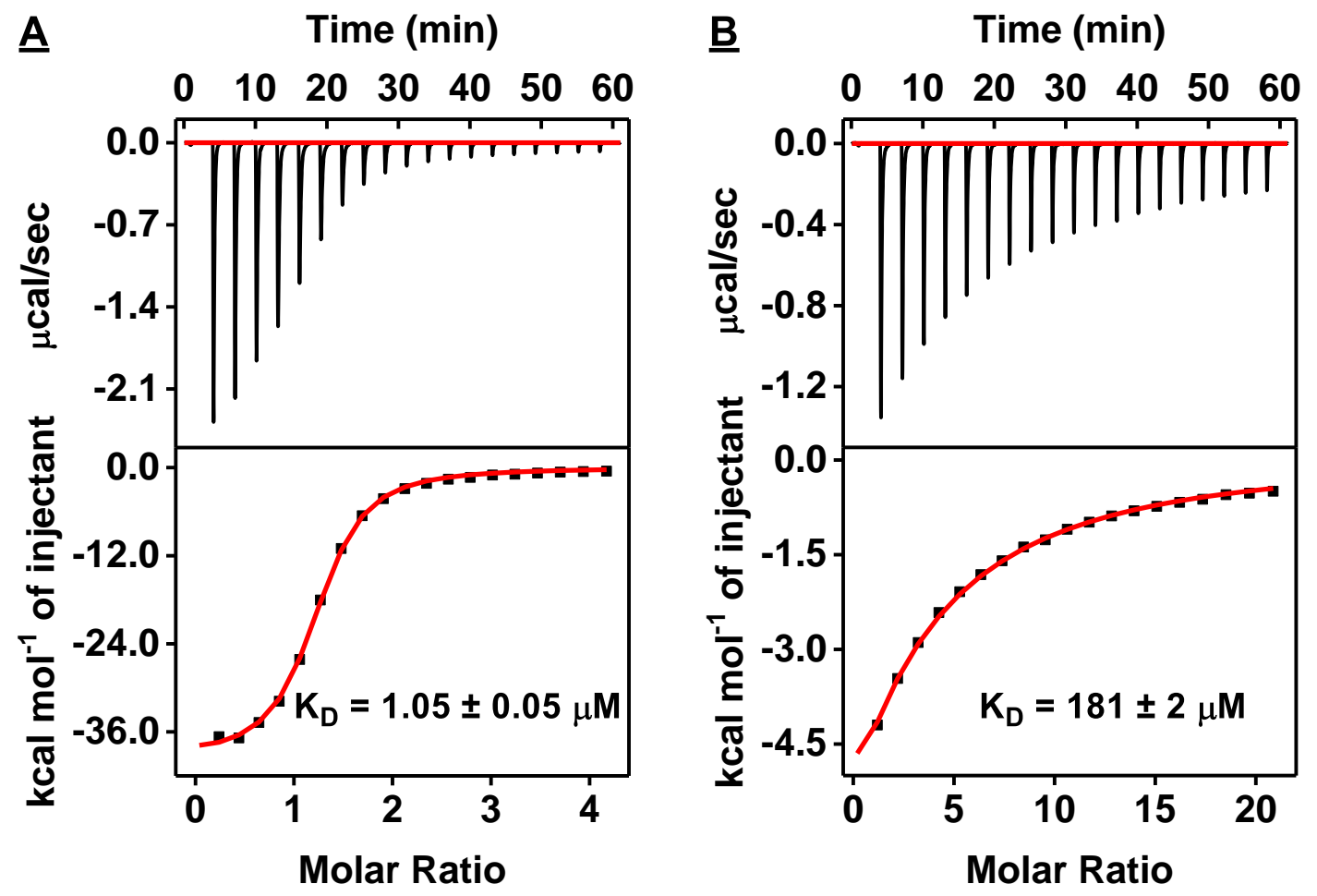

Figure S13. Characterization of the target-binding affinity of SCA1.1-40 using ITC. Top panels present raw data showing the heat generated from each titration of (A) (-)-MDPV and (B) (+)MDPV to SCA1.1-40, while bottom panels show the integrated heat of each titration after correcting for dilution heat of the titrant. ITC data were fitted using a single-site model. 

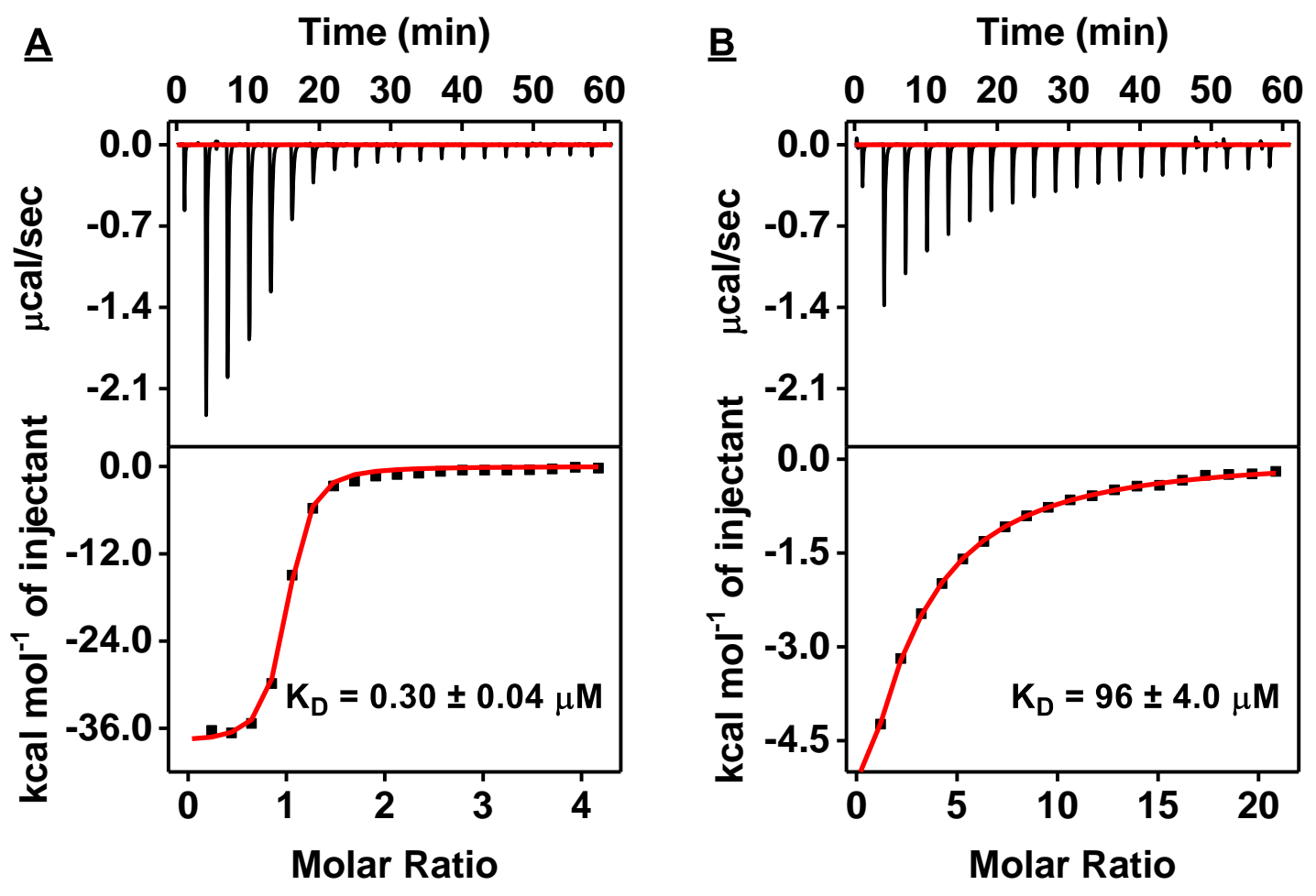

Figure S14. Characterization of the target-binding affinity of SCA2.1-34 using ITC. Top panels present raw data showing the heat generated from each titration of (A) (-)-MDPV and (B) (+)MDPV to SCA2.1-34, while bottom panels show the integrated heat of each titration after correcting for dilution heat of the titrant. ITC data were fitted using a single-site model. 

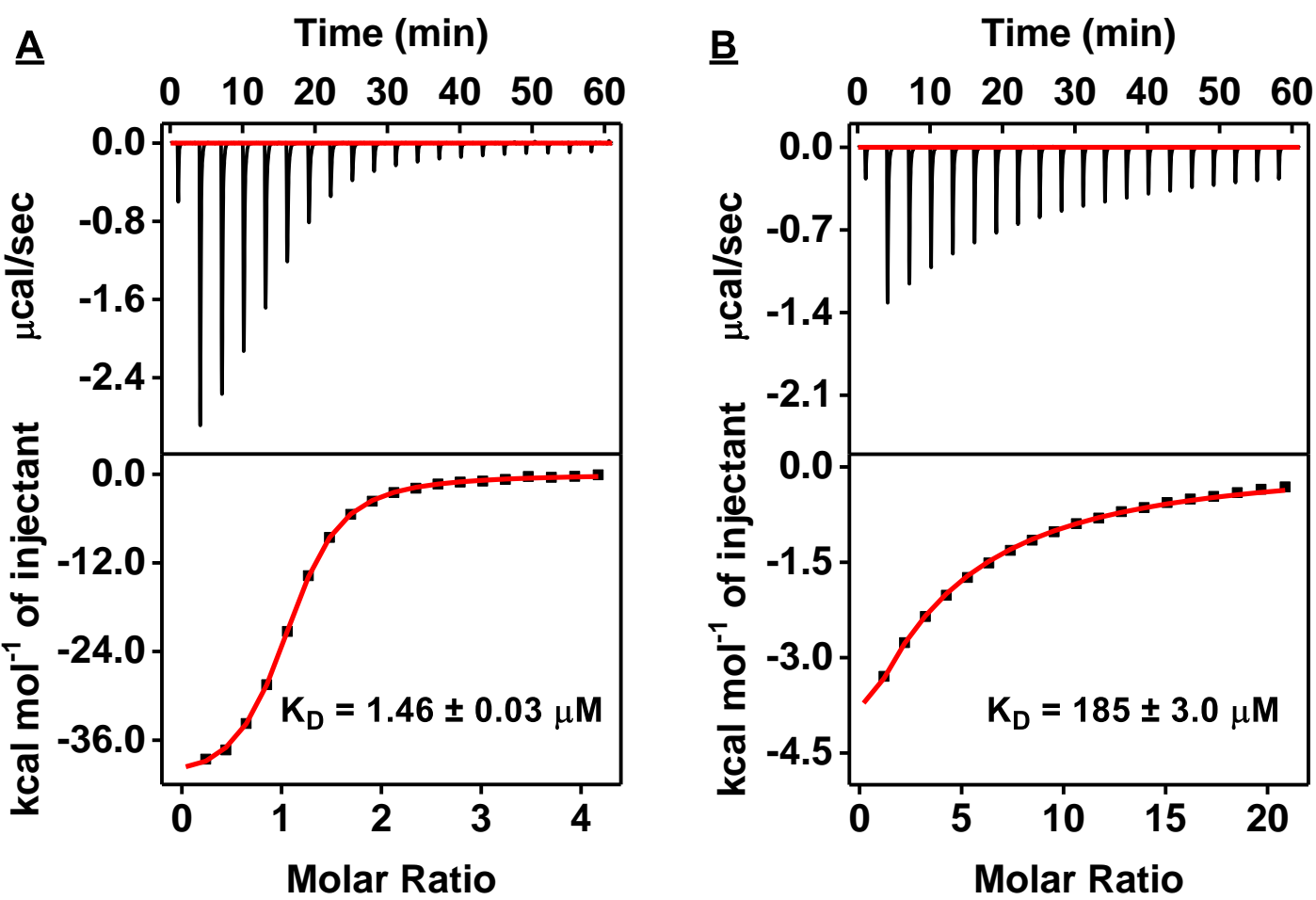

Figure S15. Characterization of the target-binding affinity of SCA1.1-34 using ITC. Top panels present raw data showing the heat generated from each titration of (A) (-)-MDPV and (B) (+)MDPV to SCA1.1-34, while bottom panels show the integrated heat of each titration after correcting for dilution heat of the titrant. ITC data were fitted using a single-site model.

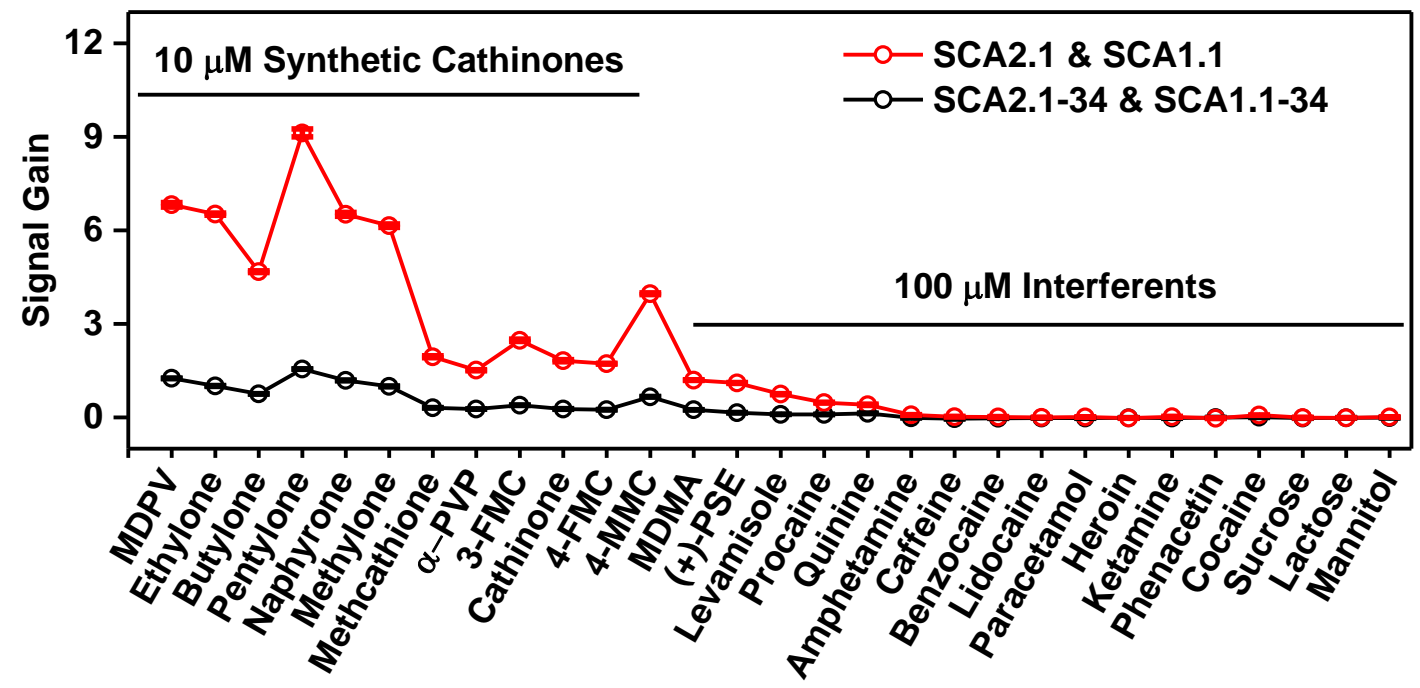

Figure S16. Signal gain of the 1:1 aptamer mixtures SCA2.1 \& SCA1.1 (red) and SCA2.1-34 \& SCA1.1-34 (black) as determined by the Cy7-displacement assay towards 12 synthetic cathinones and 17 interferents at concentrations of $10 \mu \mathrm{M}$ and $100 \mu \mathrm{M}$, respectively. Error bars represent the standard deviation of three individual experiments. 

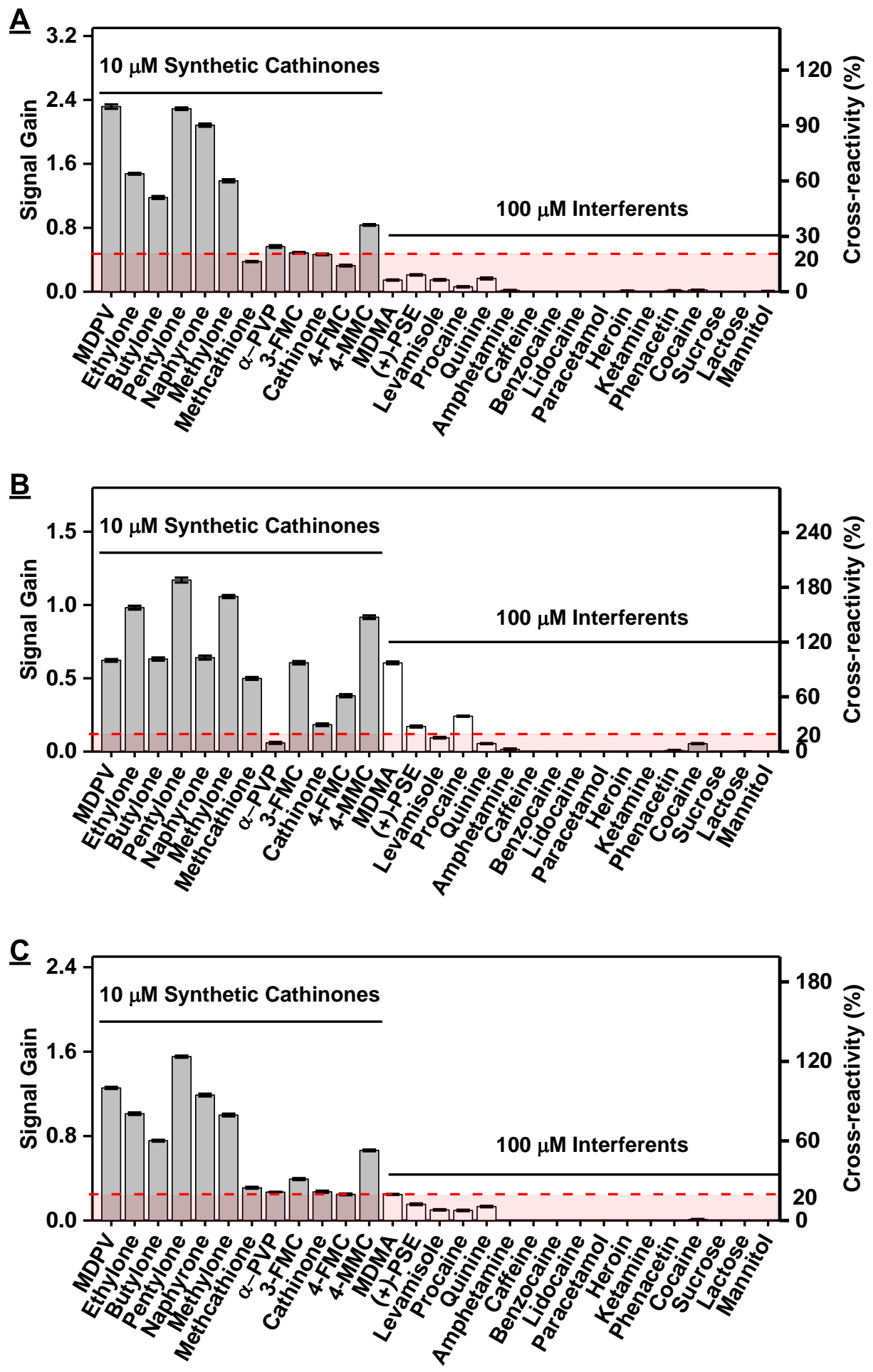

Figure S17. Signal gain and cross-reactivity from colorimetric Cy7-displacement assays performed with (A) SCA2.1-34 alone (B) SCA1.1-34 alone or (C) the mixture of SCA2.1-34 + SCA1.1-34 against 12 synthetic cathinones (gray) at a concentration of $10 \mu \mathrm{M}$ and 17 interferents (white) at a concentration of $100 \mu \mathrm{M}$. The cut-off for $20 \%$ cross-reactivity is marked in red. Error bars represent the standard deviation of three measurements. 

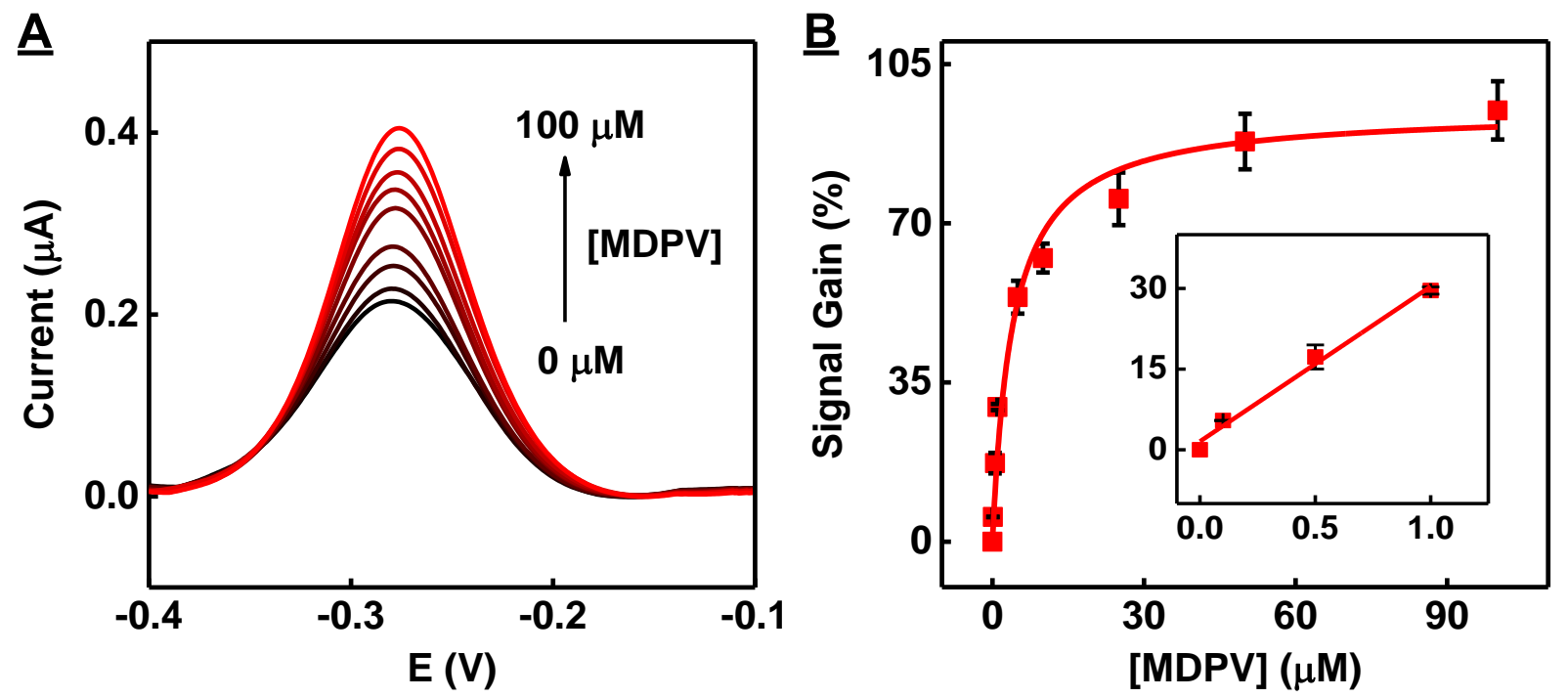

$\underline{\mathbf{C}}$

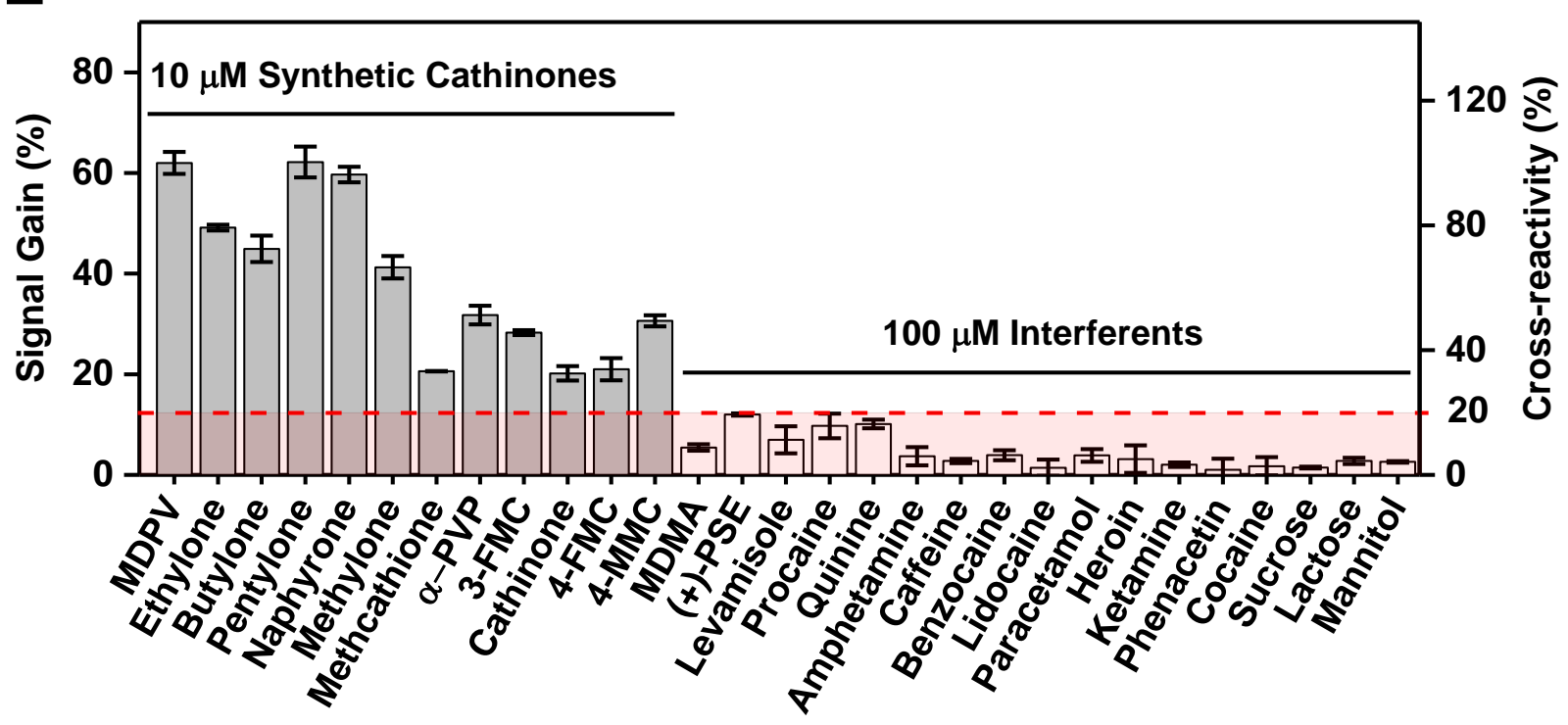

Figure S18. Performance of an E-AB sensor modified with SCA2.1-34-MB alone. (A) Squarewave voltammetry spectra collected at various concentrations of MDPV. (B) Calibration curve derived from squarewave voltammetry spectra. (C) Sensor cross-reactivity for 12 synthetic cathinones (gray) at a concentration of $10 \mu \mathrm{M}$ and 17 interferents (white) at a concentration of 100 $\mu \mathrm{M}$. Cross-reactivity is calculated relative to the signal gain produced by $10 \mu \mathrm{M}$ MDPV. The cutoff for $20 \%$ cross-reactivity is marked in red. Error bars represent the standard deviation of measurements from three different $\mathrm{E}-\mathrm{AB}$ sensors. 

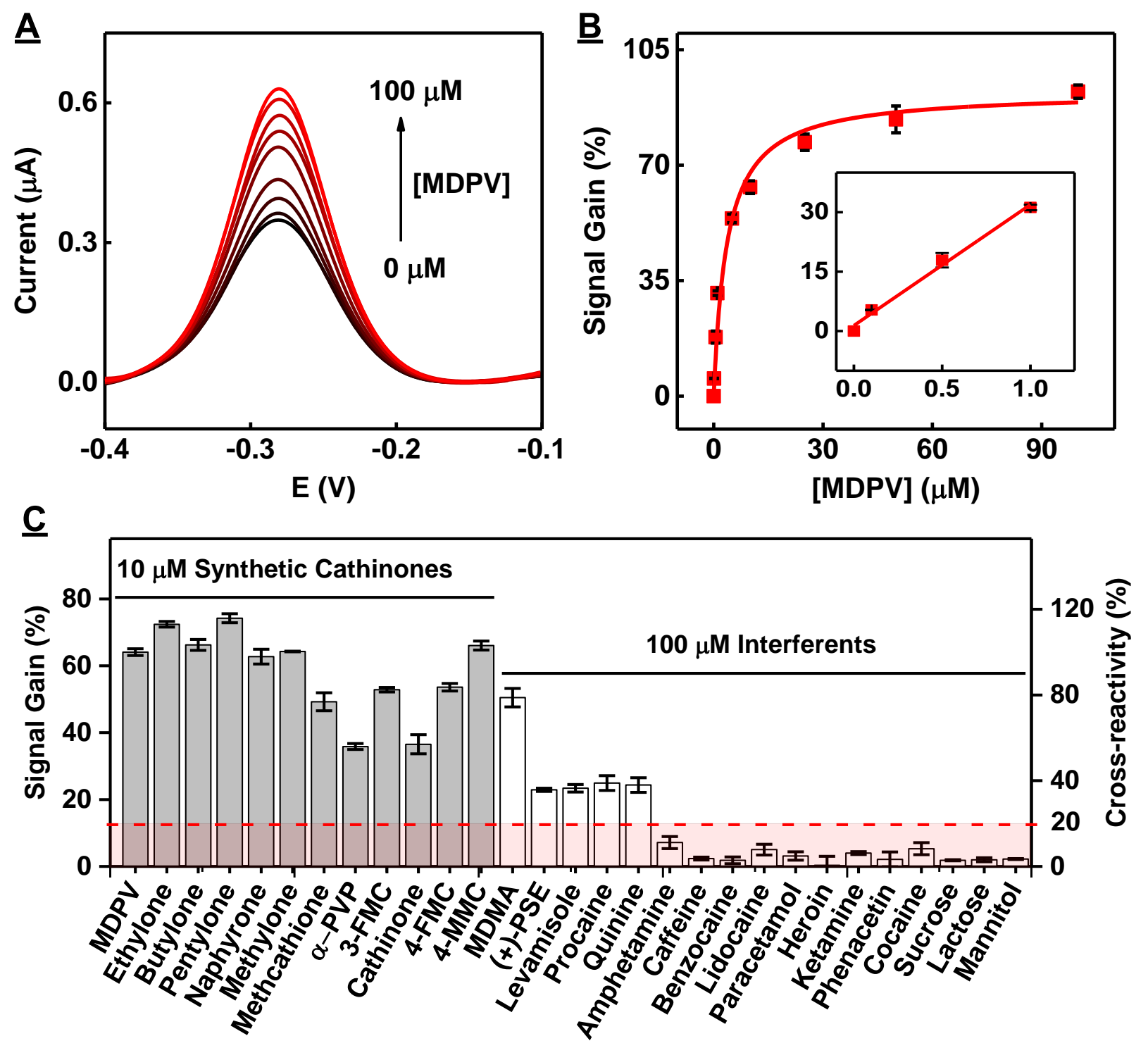

Figure S19. Performance of an E-AB sensor modified with SCA1.1-34-MB alone. (A) Squarewave voltammetry spectra collected at various concentrations of MDPV. (B) Calibration curve derived from squarewave voltammetry spectra. (C) Sensor cross-reactivity for 12 synthetic cathinones (gray) at a concentration of $10 \mu \mathrm{M}$ and 17 interferents (white) at a concentration of 100 $\mu \mathrm{M}$. Cross-reactivity is calculated relative to the signal gain produced by $10 \mu \mathrm{M}$ MDPV. The cutoff for $20 \%$ cross-reactivity is marked in red. Error bars represent the standard deviation of measurements from three different $\mathrm{E}-\mathrm{AB}$ sensors. 

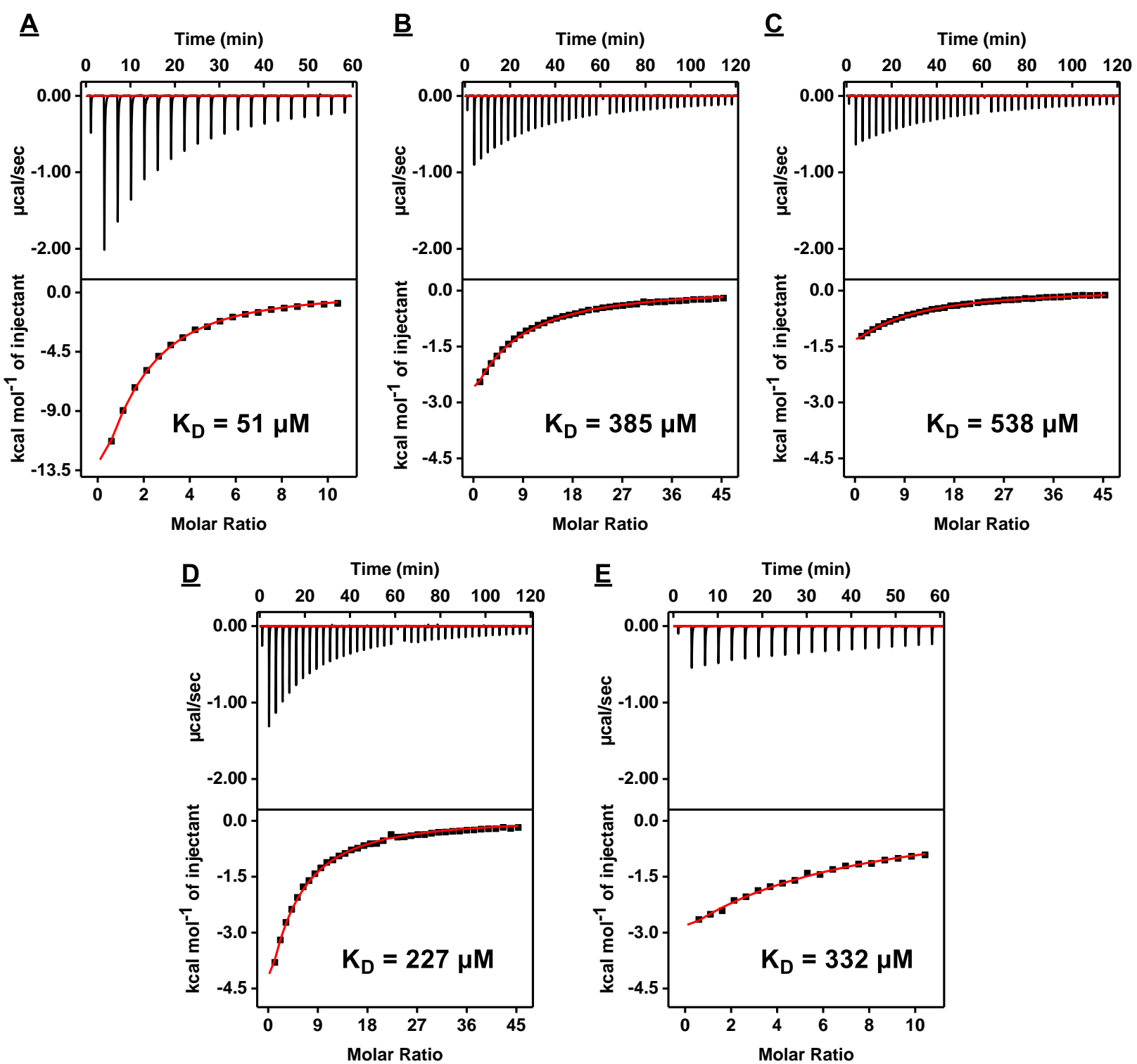

Figure S20. Characterization of the interferent-binding affinity of SCA1.1-34 using ITC. Top panels present raw data showing the heat generated from each titration of (A) MDMA, (B) (+)pseudoephedrine, (C) levamisole, (D) procaine, and (E) quinine to SCA1.1, while bottom panels show the integrated heat of each titration after correcting for dilution heat of the titrant. ITC data were fitted using a single-site model. 


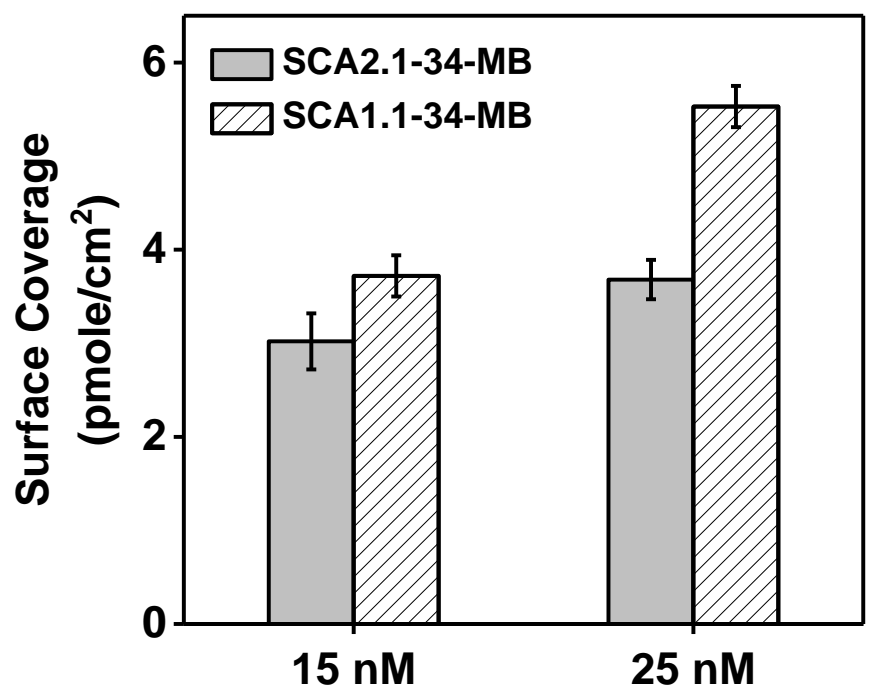

Figure S21. Comparison of surface coverages of electrodes modified with SCA2.1-34-MB or SCA1.1-34-MB alone at concentrations of $15 \mathrm{nM}$ and $25 \mathrm{nM}$ aptamer. Error bars represent the standard deviation of measurements from three different $\mathrm{E}-\mathrm{AB}$ sensors. 

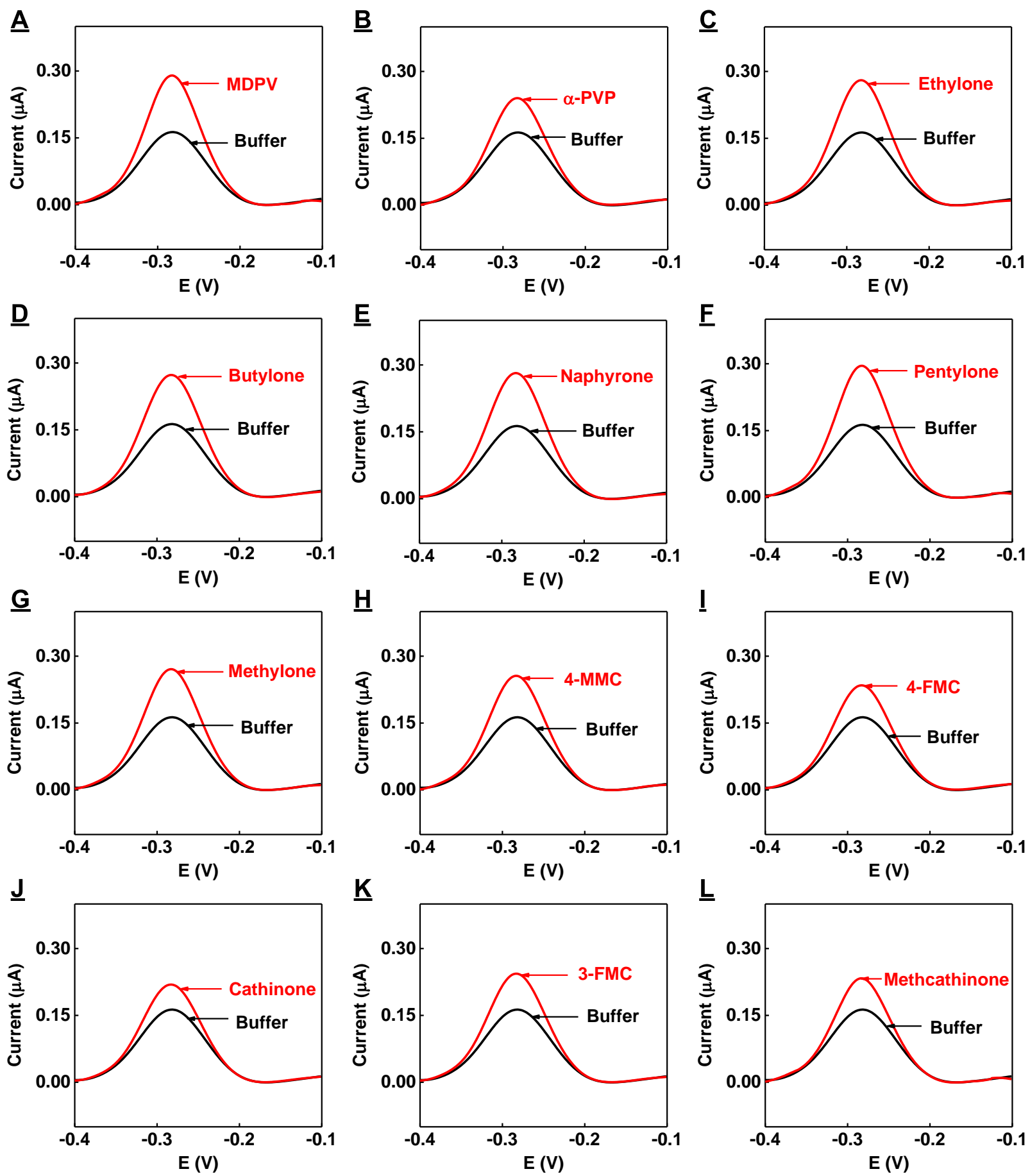

Figure S22. Squarewave voltammetry spectra collected in the absence (black) and presence (red) of 12 synthetic cathinones at a concentration of $10 \mu \mathrm{M}$ using the dual-aptamer-modified E-AB sensor. 
$\underline{\mathbf{A}}$

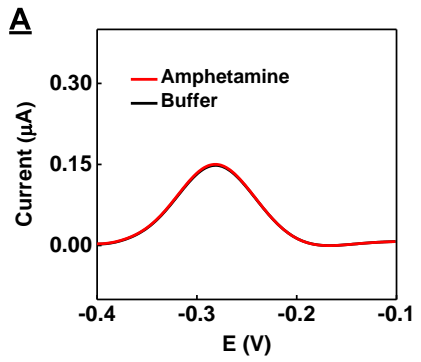

$\underline{\mathbf{D}}$

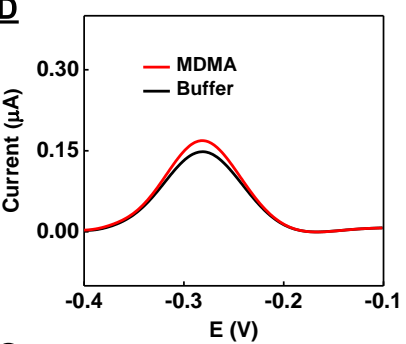

$\underline{\mathbf{G}}$

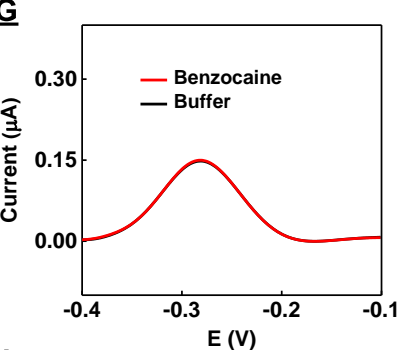

$\underline{\mathbf{J}}$
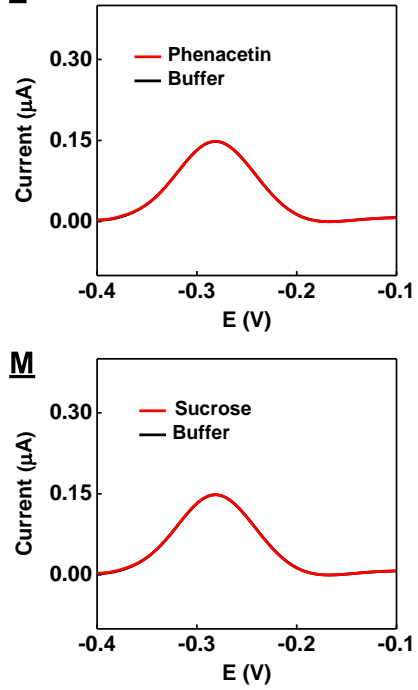
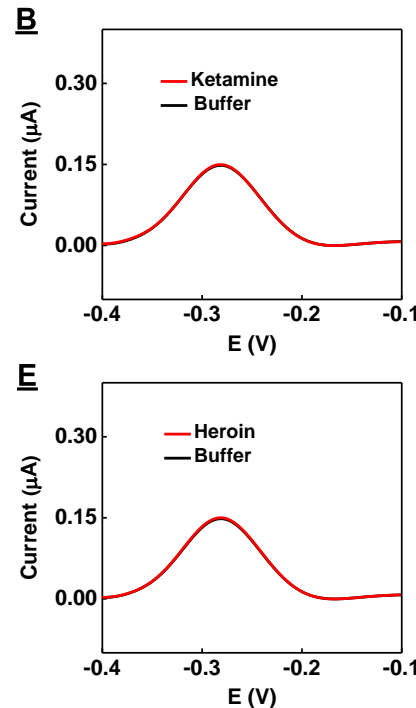

$\underline{\mathrm{H}}$

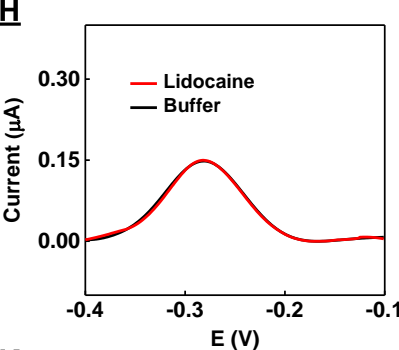

$\underline{\mathbf{K}}$
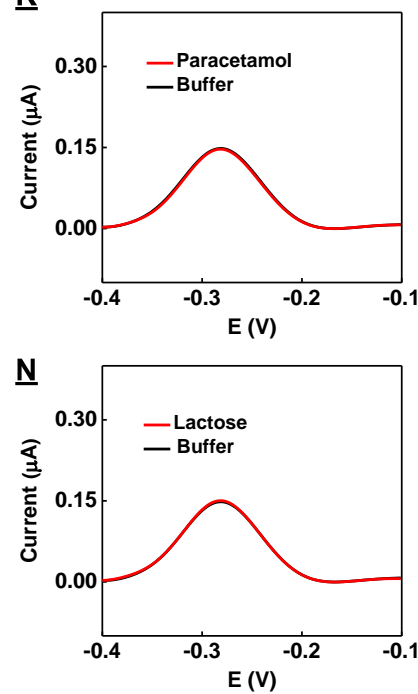
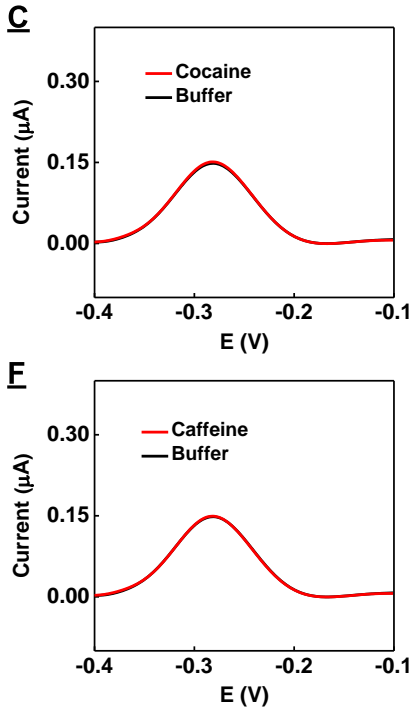

1
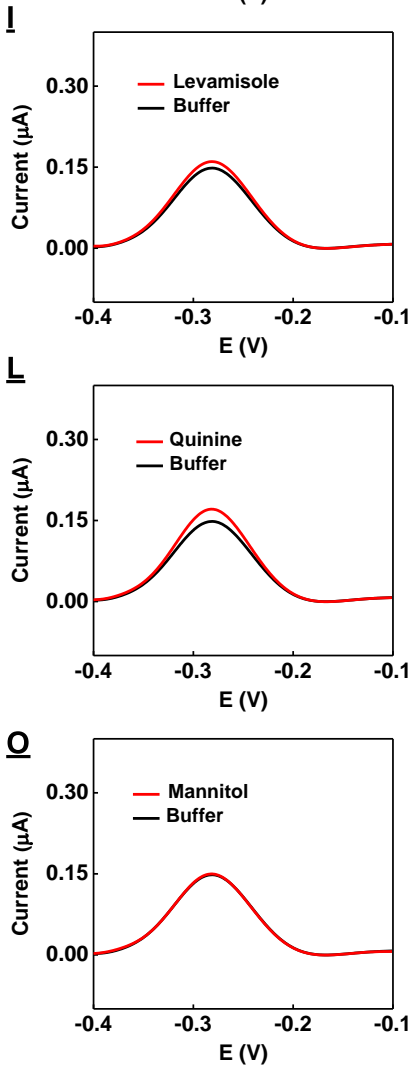
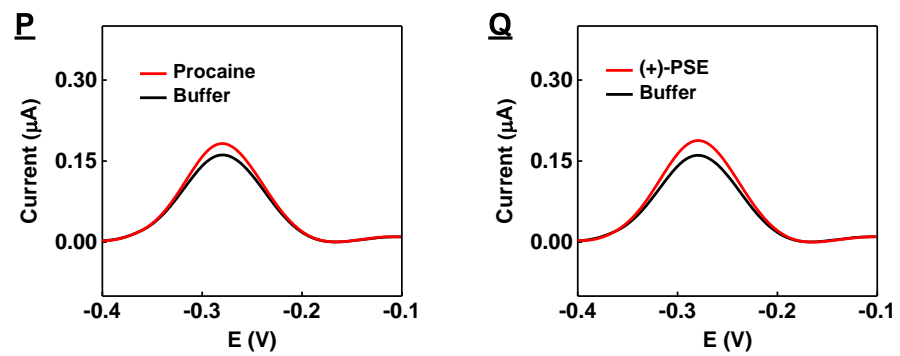

Figure S23. Squarewave voltammetry spectra collected in the absence (black) and presence (red) of 17 interferents at a concentration of $100 \mu \mathrm{M}$ using the dual-aptamer-modified $\mathrm{E}-\mathrm{AB}$ sensor. 


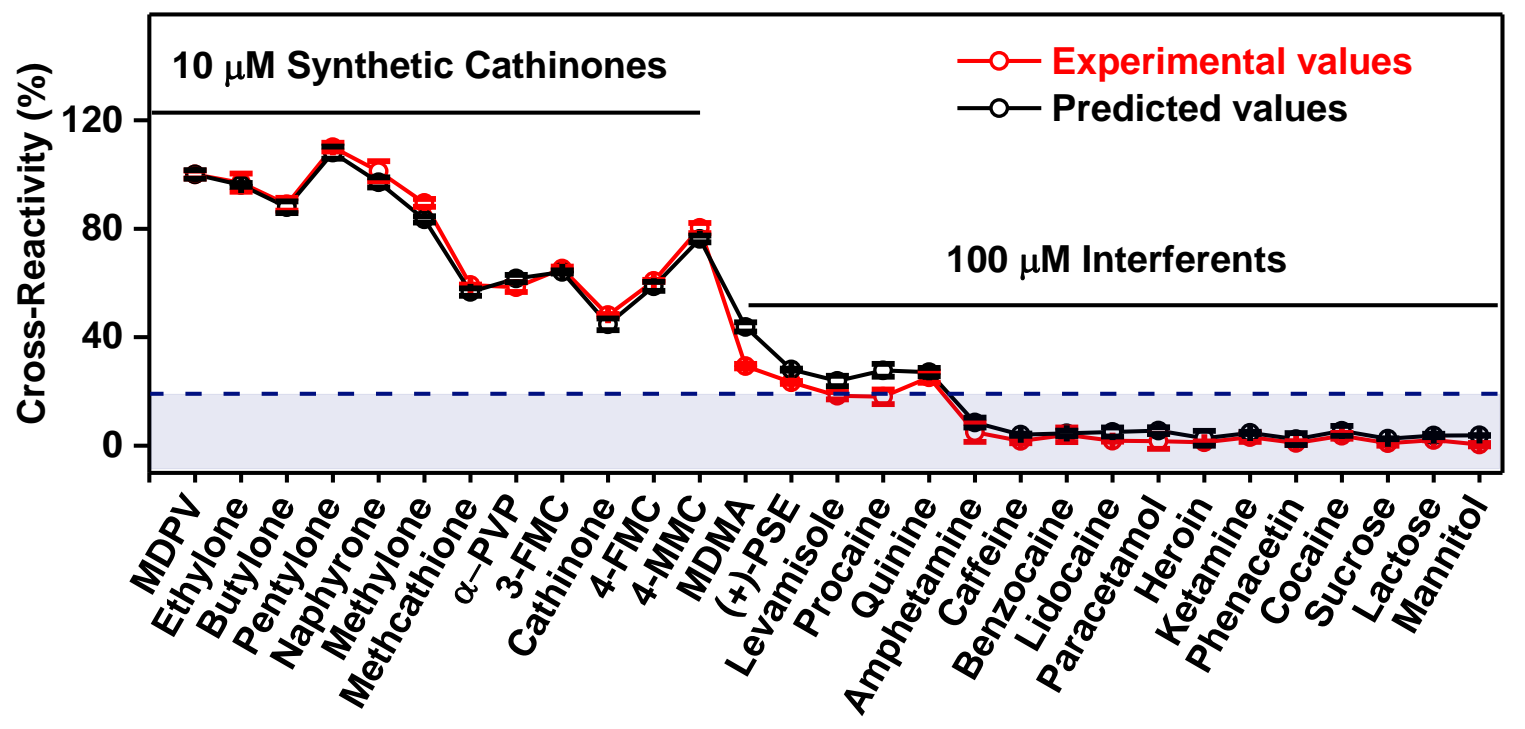

Figure S24. Experimental (red) and predicted (black) cross-reactivity of SCA2.1-34 and SCA1.1-34 mixtures using E-AB sensors with 12 synthetic cathinones and 17 interferents at concentrations of $10 \mu \mathrm{M}$ and $100 \mu \mathrm{M}$, respectively. The cut-off for $20 \%$ cross-reactivity is marked in blue. Error bars represent the standard deviation of three individual experiments. 

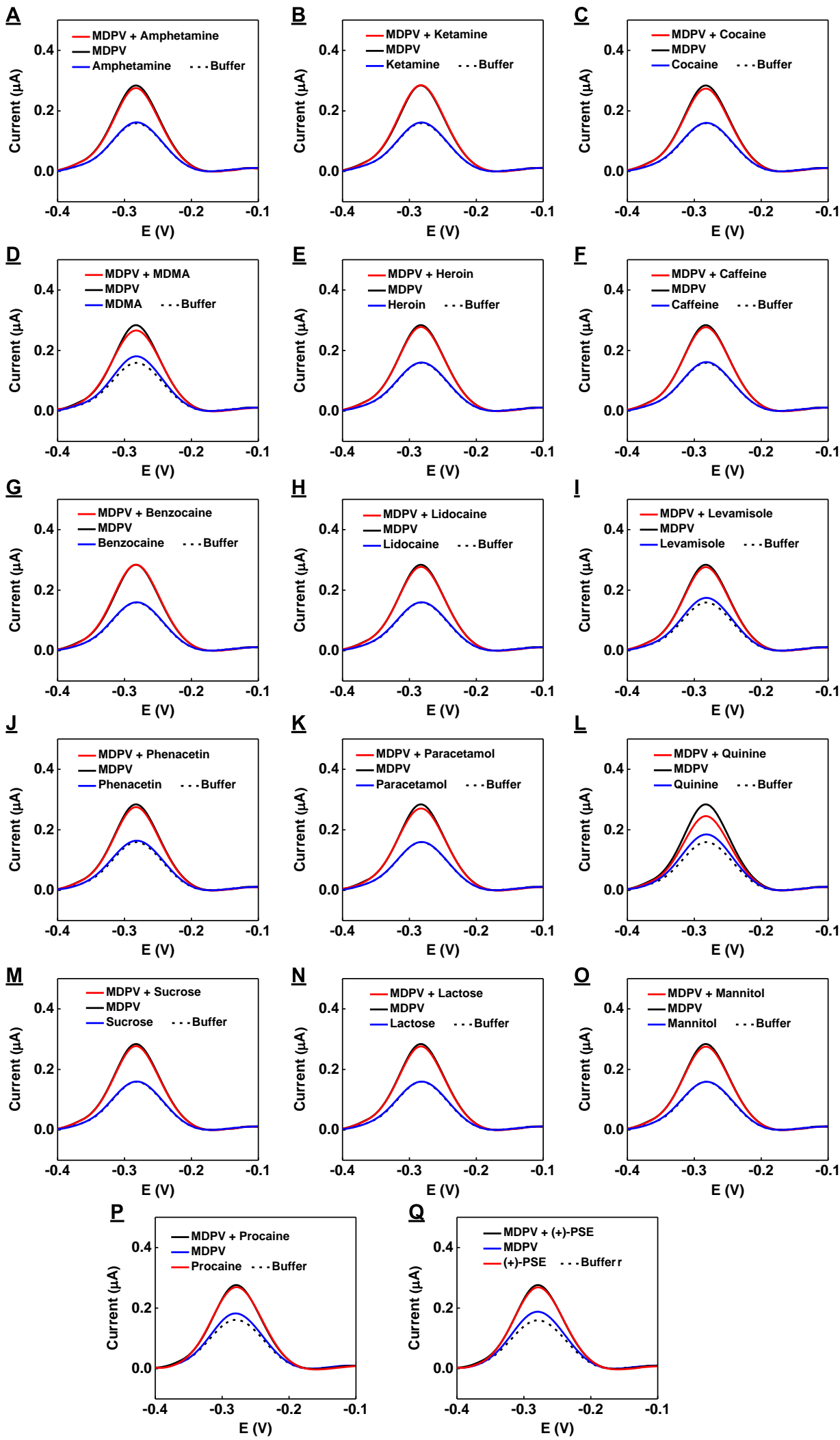

Figure S25. Squarewave voltammetry spectra collected in buffer (dashed), with $100 \mu \mathrm{M}$ of 17 different interferents (blue), $10 \mu \mathrm{M}$ MDPV (black), or a binary mixture of $10 \mu \mathrm{M} \mathrm{MDPV}$ and 100 $\mu \mathrm{M}$ of the various interferents (red) using a dual-aptamer-modified $\mathrm{E}-\mathrm{AB}$ sensor. 


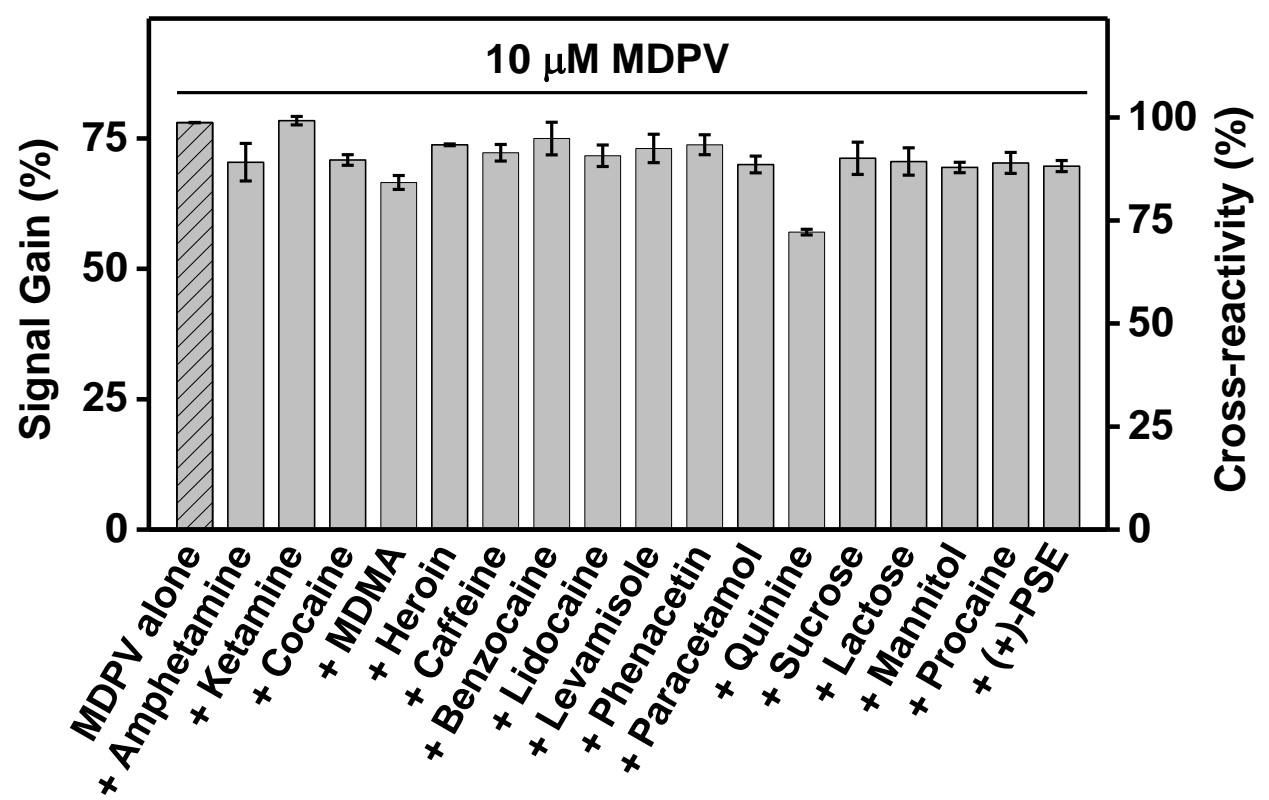

Figure S26. Signal gain and cross-reactivity from binary mixtures of $10 \mu \mathrm{M}$ MDPV and 17 different interferents at a concentration of $100 \mu \mathrm{M}$ using the dual-aptamer-modified E-AB sensor.

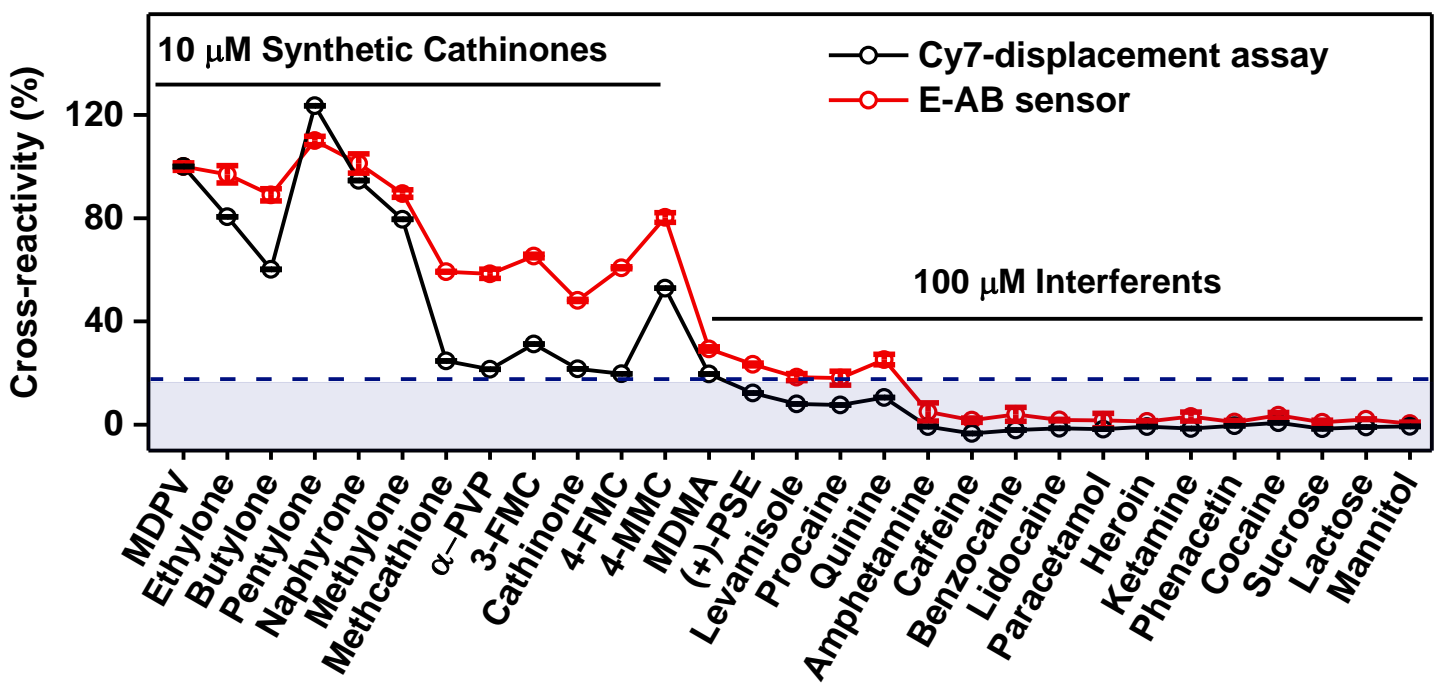

Figure S27. Cross-reactivity of the truncated mixture of SCA2.1-34 and SCA1.1-34 in the Cy7displacement assay and an $\mathrm{E}-\mathrm{AB}$ sensor with 12 synthetic cathinones and 17 interferents at concentrations of $10 \mu \mathrm{M}$ and $100 \mu \mathrm{M}$, respectively. The cut-off for $20 \%$ cross-reactivity is marked in blue. Error bars represent the standard deviation of three individual experiments. 